Portland State University

PDXScholar

$1-1-2010$

\title{
Family relationship quality and acculturation: Examination of their relationship among Latino adolescent sexual offenders
}

Lindsey B. Patterson

Portland State University

Follow this and additional works at: https://pdxscholar.library.pdx.edu/open_access_etds Let us know how access to this document benefits you.

Recommended Citation

Patterson, Lindsey B., "Family relationship quality and acculturation: Examination of their relationship among Latino adolescent sexual offenders" (2010). Dissertations and Theses. Paper 165.

https://doi.org/10.15760/etd.165

This Thesis is brought to you for free and open access. It has been accepted for inclusion in Dissertations and Theses by an authorized administrator of PDXScholar. Please contact us if we can make this document more accessible: pdxscholar@pdx.edu. 
Family Relationship Quality and Acculturation: Examination of Their Relationship Among Latino Adolescent Sexual Offenders

by

Lindsey B. Patterson

A thesis submitted in partial fulfillment of the requirements for the degree of

\author{
Master of Science \\ in \\ Psychology
}

Portland State University

2010 


\begin{abstract}
Research on child sexual abuse (CSA), from the perspective of the perpetrator, has been conducted to better inform intervention and prevention programs. Although information from perpetrators can be beneficial for these programs, much of the research is limited by the lack of diversity of sample populations of sex offenders. Moreover, potentially distinct variables relevant to specific populations (e.g., Latinos) have not been thoroughly studied in relation to CSA. To better understand the perpetration of CSA on variables that may be of particular concern to Latinos (i.e., relationship quality in familial supervision and acculturation strategies), the purpose of the present study was to investigate the relationships between supervisor relationship quality, acculturation, and adolescent group membership (i.e., juvenile sex offender - JSO and juvenile comparison - JC). It was hypothesized that Latinos who are assimilated or marginalized are more likely to belong to the JSO group than the JC group. Further, Latino adolescents characterized by an integrated or separated acculturation strategy are more likely to be affiliated with JC group than the JSO group. It was also hypothesized that participants' relationship with their familial supervisor will predict adolescent membership and that acculturation will mediate this relationship. Results for both hypotheses were inconclusive. The probability of using a specific acculturation strategy was not statistically different for either adolescent group. The relationship between supervisor relationship quality and juvenile group membership was non-significant; therefore, the meditational role of acculturation could not be assessed. Despite non-significant results, some relationships were in the predicted direction. Further research, using a larger
\end{abstract}


sample size with more complete data is recommended. Suggestions for other design improvements are also provided. 


\section{Acknowledgements}

I would like to thank my advisor, Dr. Keith Kaufman, for his encouragement and support throughout the thesis process. I would also like to thank my other committee members, Dr. Yves Labissiere and Dr. Eric Mankowski, for their participation on the committee and for their thoughtful suggestions on my thesis. Finally, I would like acknowledge Dr. Todd Bodner, whose statistical guidance was much appreciated. 


\section{TABLE OF CONTENTS}

ABSTRACT

ACKNOWLEDGEMENTS

LIST OF FIGURES $\quad$ v

LIST OF TABLES vi

$\begin{array}{ll}\text { INTRODUCTION } & 1\end{array}$

$\begin{array}{ll}\text { METHODS } & 26\end{array}$

$\begin{array}{ll}\text { RESULTS } & 31\end{array}$

$\begin{array}{ll}\text { DISCUSSION } & 56\end{array}$

$\begin{array}{ll}\text { LIMITATIONS } & 65\end{array}$

IMPLICATIONS AND FUTURE DIRECTIONS 70

$\begin{array}{ll}\text { FIGURES } & 74\end{array}$

$\begin{array}{ll}\text { TABLES } & 84\end{array}$

$\begin{array}{ll}\text { REFERENCES } & 96\end{array}$

$\begin{array}{lr}\text { APPENDICES } & 112\end{array}$

Appendix A. SQ Part 1, Questions 3a-s 112

Appendix B. SQ Part 1, Questions 30a-g 114

Appendix C. SQ Part 1, Questions 32a-n 115

Appendix D. SQ Part 1, Questions 31a-f 117

Appendix E. MEIM and Other-group Orientation Scale 118

Appendix F. Language Usage Scales 120 


\section{LIST OF FIGURES}

$\begin{array}{ll}\text { Figure 1. } & 74\end{array}$

Multidimensional Model of Acculturation

Figure 2.

Mediated Model for Hypothesis 2

Figure 3.

Line Graph of Interaction between Grade Level Completion and Ethnicity

Figure 4.

Factor Model for Supervisor Relationship Quality

Figure 5.

Factor Model for Supervisor Relationship Quality Improvement for Latinos

Figure 6.

Factor Model for English Language Usage

Figure 7.

Factor Model for Spanish Language Usage

Figure 8.

Factor Model for MEIM and Other-group Orientation

Figure 9.

Dendogram for Acculturation Strategy

Figure 10.

Bar Chart of Group Assignments for Hypothesis 1 


\section{LIST OF TABLES}

Table 1.

Participants' Group Affiliation and Self-Reported Ethnicity

Table 2.

Frequencies of Participants from Each Ethnic Group by State

Table 3.

Sample Sizes for Analyses

Table 4.

Mean Age (SD) (Group Affiliation X

Self-Reported Ethnicity)

Table 5.

Percentage of Top 3 Family Members Providing Supervision (Group X Ethnicity)

Table 6.

Internal Reliabilities (i.e., Internal Consistencies)

Table 7.

Correlation Matrix for MEIM and Other-group Orientation Factors from EFA

Table 8.

Correlation Matrix of Six Acculturation Factors

Table 9.

92

Means and Standard Deviations of Clusters on Cluster Variables 
Table 10.

Post hoc ANOVA Results - Significance of Mean Cluster Differences Between Cluster Variables

Table 11.

Subsample Sizes of Acculturation Strategies from Median Splits

Table 12.

Results from the First Step of the Mediation Analysis 


\section{Introduction}

\section{Child Sexual Abuse (CSA)}

It is widely established that child sexual abuse (CSA) is a serious national and international problem. CSA has consistently been defined as exposing a child to sexual activity, including fondling, kissing, rape, or exposure to other sexual content (Centers for Disease Control [CDC], 2008). No child is immune to CSA; it transcends all racial/ethnic backgrounds and socioeconomic statuses. Since the early 1990's, the number of reported cases of CSA has been on the decline (Jones \& Finkelhor, 2001), but CSA is still a significant problem. CSA affects between 75,000 and 300,000 children and families within the United States each year (Jones \& Finkelhor, 2001; Murphy, 2002;

U.S. Department of Health \& Human Services, 2006). These statistics are mere estimates due to the fact that many cases of CSA remain unreported (Jones \& Finkelhor, 2001; Paolucci, 2001).

Under-reporting of sexual offenses, especially by juvenile offenders, is influenced by multiple factors. Some reports of sexual offense incidence depend on arrest rates. Using arrest rates is a conservative method of estimating incidence since sexual offenders are not caught or reported on the majority of offenses. This data does not necessarily include cases known to professionals or treatment facilities across the country; instead, these statistics are based on police reports and other judicial resources (Finkelhor, 1994). Arrest rates also prove to be inaccurate because many offenders commit more sexual crimes than the ones for which they are arrested. In fact, it has been suggested that the ratio between actual offenses discovered through self-report and arrest rates for sexual 
offense is 25:1 (Elliott, Huizinga, \& Morse, 1985). Another explanation for under reporting is that CSA leaves long-term scars for victims, families, and communities (Finkelhor \& Browne, 1985; Fontes, 2007; Paolucci, Genuis, Violato, 2001). Since many sex offenses are intrafamilial (U.S. Department of Health \& Human Services, 2006), the lives of the victim(s) and perpetrator are intertwined. As a result, it may be extremely difficult for families to report the offender, let alone acknowledge and cope with the sexual offense.

CSA occurs within a variety of communities, but the majority of CSA victim literature focuses on only one segment of the population, middle-class, White college students. Within the current literature, even on the dominant, White population, the statistics on incidence of CSA are inconsistent. Research on CSA within minority populations, especially within Latino $^{1}$ communities, is understudied (Bacigalupe, 2001; Fontes, Cruz, \& Tabachnick, 2001). Inconsistencies in the statistics on CSA in the dominant, White population are magnified in the few studies that have taken ethnicity into consideration (Bacigalupe, 2001).

In 2006, the U.S. Department of Health and Human Services conducted an extensive study on child maltreatment. Of the 55,550 reports of CSA in the U.S., $54.2 \%$ of the victims were Caucasian children, $17.9 \%$ were African-American, and $17.7 \%$ were Latino (U.S. Department of Human Services, 2006). Although some reports state that the number of cases of CSA are twice as high for Whites compared to minority ethnicities,

\footnotetext{
${ }^{1}$ Some literature uses the terms 'Latino' and 'Hispanic' interchangeably. However, this paper will only use 'Latino' as it connotes a specific origin of locality (i.e., Latin America) and is preferred by Latinos in the U.S. and in Latin America (Alcoff, 2005). For a detailed discussion of the difference between 'Latino' and 'Hispanic,' refer to Alcoff (2005).
} 
other research indicates that there is no difference in the prevalence of CSA between minority and majority populations (Latinos at $27.1 \%$ and Caucasians at $33.1 \%$; Arroyo, Simpson, \& Aragón, 1997). In contrast, some investigations report that minority communities experience more CSA than White Americans. For example, Ullman and Filipas (2005) found that prevalence of CSA among the African-American community (40.3\%) greatly out numbered that in the Latino (33.3\%) and Caucasian $(25.5 \%)$ communities. These discrepancies may be a result of varying definitions of CSA, measurement approaches, sample populations, policy changes, and attitude changes surrounding CSA cases (Jones \& Finkelhor, 2001; Paolucci et al., 2001). For instance, studies by Arroyo et al. (1997) and Ullman and Filipas (2005) used a limited sample of female college students and relied completely on self-report. Data developed by the U.S. Department of Health and Human Services often originate from reports made by Child Protective Services and other government organizations. Due to these limitations, existing statistics can only be utilized as an estimate of the true rates of CSA incidence among these populations.

Despite these inconsistencies, it is virtually certain that CSA will have some effect on each of its victims. Long-term effects of CSA on child victims have included depression (Hinson, Koverola, \& Morahan, 2002; Paolucci et al., 2001; Sanders-Phillips, Moisan, Wadlington, Morgan, \& English, 1995; \& Ullman \& Filipas, 1995), suicide or suicidal ideation (Paolucci et al., 2001), PTSD (Andrés-Hyman, Cott, \& Gold, 2004), eating disorders (Cachelin, Schug, Juarez, \& Monreal, 2005), and poor academic performance (Paolucci et al., 2001). Research on the effects of CSA on children and 
families have not investigated how the experience of CSA by minority populations differs from the experiences of the White majority. However, it has been suggested that the effects of CSA victimization are independent of race (Arrellano, Kuhn, \& Chávez, 1997). Although race may not play a role in the reporting or experience of CSA, it is possible that cultural elements inherent in various ethnic groups influence the perpetration and victimization of CSA. For instance, it has been suggested that some communities may have better support systems or coping mechanisms as a result of cultural values (Bacigalupe, 2001). Therefore, cultural values are important to examine when investigating the impacts of the serious social issue that is CSA.

Although much of the literature discusses the negative impacts that CSA has on children, less research has been conducted on the perpetration of CSA. Despite the lack of information on the perpetration of CSA in some areas of the field (e.g., differences in perpetration between majority and minority populations), the literature does define general typologies of perpetrators depending on sex and age. Perpetrators of CSA include adults, adolescents, and even children that are between 3 to 5 years the victim's senior (Centers for Disease Control [CDC], 2008; Murphy, 2002). Research has shown that female and male perpetrators are distinct (Johnson, 1988), and the majority of offending is perpetrated by males (Bureau of Justice Statistics, 2007; Davis \& Leitenberg, 1987). Historically, a great deal of attention has focused on adults as the primary perpetrators of CSA (Becker \& Abel, 1985; Davis \& Leitenberg, 1987; Groth \& Loredo, 1981; Starzyk \& Marshall, 2003; Veneziano \& Veneziano, 2002). However, literature indicates that many adults begin offending during their adolescent years (Abel, Osborn, 
\& Twigg, 1993). In fact, juveniles have been found to account for $20-50 \%$ of all child sexual offenders (Barbaree \& Marshall, 2006; Davis \& Leitenberg, 1987; Groth \& Loredo, 1981; Knight \& Prentky, 1993). Other researchers have asserted that adolescents are a unique population to study because they are in the processes of defining their identity and sexual self (Bischof, Stith, \& Wilson, 1992; Groth \& Loredo, 1981; Hunter \& Becker, 1994; Knight \& Prentky, 1993). Although sexual development takes place throughout the lifespan, adolescence is a time when many changes and influences converge. Adolescents can experience changes in physical appearance, peer pressures, changing definitions of identity and autonomy, changing relationships with friends and family, and media persuasion (Bukowski, Sippola, \& Brender, 1993). Navigating these changes and defining the (sexual) self is an ongoing process (Bancroft, 2006; Bukowski et al., 1993). Clearly, the effects of intervention with problematic sexual behavior may have different consequences when dealing with adolescents (Groth \& Loredo, 1981). This is reflected in the significantly lower recidivism rates exhibited by juveniles as compared to adult sex offenders (Knight \& Prentky, 1993). The dynamic nature of adolescents' sexual malleability points to the greater opportunity for successful treatment interventions and underscores the reasons for a focus on male juvenile sex offenders in this study. In the developmental literature, the terms 'adolescent' and 'juvenile' have slightly different meanings. 'Adolescent' refers to the developmental transition between childhood and adulthood whereas 'juvenile' refers to a specific legal time period for an individual between the ages of 13 and 18 (Barbaree, Hudson, \& Seto, 1993). Literature on juvenile sex offenders uses these terms interchangeably (Barbaree et al., 1993; 
Langton \& Barbaree, 2006). The present study mirrors research literature on juvenile sex offenders using the terms 'adolescent' and 'juvenile' interchangeably.

The principle concern in the present study is to examine the relationships between potentially distinct family relationships and acculturation strategies and perpetration of CSA by juvenile offenders. More specifically, this study focuses on the Latino community, as an example of an understudied population within the CSA literature. The subsequent review of the literature begins by framing the perpetration of CSA in terms of family relationships. The literature review then explores acculturation as a key contextual foundation for the investigation of adolescent Latinos in the U.S. Briefly, acculturation is defined as the process of cultural learning as two or more cultures come into consistent contact. The relationship between acculturation and CSA will also be discussed. Finally, the review will conclude with an examination of the relationship between family relationships and acculturation and their association with the perpetration of CSA. A critique of the literature will follow which will highlight the need to explore the relationship between family relationships and acculturation in juvenile sex offenders.

\section{Population Specific Research on Latinos}

The United States is home to a plethora of ethnic groups that maintain numerous and distinct cultural heritages. Mio, Barker, and Tumambing (2009) contend that the U.S. is a multicultural society; therefore, practioners and researchers alike need to understand and examine social problems using a multicultural perspective. They define this perspective as the "study of behavior, cognition, and affect in many cultures" (p. 4). 
Before comparing the impact of different cultures on individuals and groups, researchers must first thoroughly understand the specific cultures in question. Population specific psychology is responsible for examining the nature of culture in unique ethnic groups as well as its impact on social problems, like CSA.

A complete understanding of the experience of CSA within diverse communities is inhibited by the narrow-minded focus of current research that ignores experiences of minority populations. This focus can be seen as the result of the strict nature of the methodology of science. Rappaport (2005) describes research as biased by those who fund it, primarily state and federal governments. Methodological conservatism (i.e., limitations on qualitative methods) and mono-disciplinary understandings of social problems are two distinct factors that limit multicultural and population specific research. The majority population, White America, has been studied extensively, while little reference to other communities, especially minority communities, is made. What seems to be missing in the literature on CSA is the idea of relativism or "contextualism" which assert that every experience can be seen only in a sociocultural context between the person and the environment (Rappaport, 1977; Trickett, 1996). Furthermore, CSA is experienced, both on the part of the victim and the perpetrator, through an ecological framework that is contingent on the community in which they live and cultural values they maintain.

Despite the fact that Latinos are currently estimated to be the largest minority group in the United States (U.S. Census Bureau, 2009), Latinos are consistently underserved and understudied in relation to many social problems including CSA 
(Bacigalupe, 2001). However, studying a population as large and diverse as all Latinos in the U.S. can be problematic. It is important to acknowledge that there is a great deal of heterogeneity when describing Latinos. The group referred to as "Latino" consists of people from many different backgrounds, ascribing to a diverse array of cultural identities (Trickett, 1996; Bacigalupe, 2001). Latinos emigrate to the U.S. from many different countries, including Mexico, Puerto Rico, Costa Rica, Ecuador, Chile, and all other Central, South American, and Caribbean countries. Immigrants from Spain and Portugal are also included in some definitions of Latino. Among and even within these countries, culture varies. In combination with the specific country of origin, Latinos represent an array of experiences here in the U.S. and have unique qualities (Bacigalupe, 2001). For example, some Latino families moved to the U.S. generations ago while others families immigrated here within the last few months. Even a basic assumption of language consistency, that Latinos primarily speak Spanish, is not consistent across all Latino communities (Bacigalupe, 2001). It is important to recognize the heterogeneity within the Latino population. At the same time, there is some value in studying Latinos as a whole.

While researchers need to be cautious in their approach to studying heterogeneous populations, there is some merit to examining CSA among Latino communities. There are broad similarities (e.g., navigating the acculturation process and oppression from dominant American society) among Latinos, and these experiences do relate to one another (Bacigalupe, 2001). More importantly, researchers need to explore the characteristics that distinguish Latinos from the dominant, White American population 
(Bacigalupe, 2001). As a minority within a country quick to exclude outsiders, Latinos often have similar experience with fragmentation or dislocation, rejection, and invalidation (Bacigalupe, 2001). For these reasons, it is important to understand CSA within the diverse Latino context as a first step in investigating cultural differences.

Although research on CSA is dominated by studies of the White populations (Arroyo, Simpson, \& Aragon, 1997), an attempt to better understand the incidence and root causes of CSA within Latino communities is developing. Championed by researchers who include Gonzalo Bacigalupe and Lisa Fontes, contextual relativism and CSA are now seen as inter-related. Literature by these researchers emphasizes the relationship between cultural differences and CSA within Latino communities. They describe CSA in terms of engendered roles in society and the family, acculturation, immigration issues, and oppression. These experiences are particularly relevant when combined with the notion that many Latinos encounter fragmentation of culture, rejection, and invalidation in the U.S. (Bacigalupe, 2001). The differences between cultural beliefs and various levels of acculturation within the U.S. create an array of experiences for Latinos. These experiences influence all parts of life, including the experience of CSA.

Although the Latino experience of social problems is heterogeneous, CSA has been recognized as a significant problem within Latino communities (Fontes et al., 2001). In their qualitative research on CSA in two diverse communities of Latinos and African Americans, Fontes, Cruz, and Tabachnick (2001) reported universal themes to Latinos describing the personal and community-based etiology of CSA. For the Latino 
community, the risk for perpetrating sexual abuse originates in "changing cultural and family factors" (Fontes et al., 2001, p. 108). Small group discussions also revealed a heavy emphasis on the family's role. Through these discussions, Latinos expressed concerns as they recognized that family has potential to house perpetrators, but it also serves as the principle support for recovery from CSA (Fontes et al., 2001). Bacigalupe's (2001) ideas about CSA support the findings by Fontes et al. (2001). He asserts that researchers and practioners "...need to consider the potential contribution of extended family members or those the family consider 'family' like godparents, friends, or distant relatives to protect children, confront perpetrators, and foster healing" (p. 174). Family is a clear and integral theme among discussions of CSA in Latino communities. This theme reflects the need to understand the perpetration of CSA through family relationships.

\section{Family Relationships and Dysfunction}

Literature on the etiology of sexual offenders has consistently pointed to family dysfunction as a principle risk factor for offending. Early childhood experiences and family relations play an essential role in the development of thoughts and behaviors of children and adolescents (Starzyk \& Marshall, 2003). Relationships with parents, caregivers, and other family members as well as negative experiences during childhood can create cognitive, behavioral, and interpersonal templates that may lead to delinquent behaviors like sexual offending (Davis \& Leitenberg, 1987; Marshall \& Marshall, 2000; Starzyk \& Marshall, 2003). Detached or poor relationships with parents, violence in the home, and sexual offenders in the extended family have all been associated with sexual 
offending in adolescence and adulthood (Starzyk \& Marshall, 2003; Veneziano \& Veneziano, 2002).

Poor relationships with parents or caregivers have been found to relate to behavioral problems, including sexual offending (Barbaree \& Langton, 2006; Starzyk \& Marshall, 2003; Veneziano \& Veneziano, 2002). Theoretical explanations of CSA have cited poor attachments to parents as an initial factor in the etiology of sexual offending (Marshall, 1993). Research studies have supported this theory. Reports from adult sex offenders indicate that perceived poor attachments with parents, especially with mothers, increase a child's vulnerability to risk factors for sexual offending (Marshall \& Mazzuco, 1995). Research on juvenile sex offenders reveals similar results. In a study of adolescent sex offenders, Friedrich and Luecke (1988) characterized a large majority $(93.75 \%)$ of the relationships between sexually aggressive male youth and their parent(s) as poor (i.e., lack of child support, a history of "scapegoating" and projection, and a history of neglect and abandonment). Poor relationships between child and parent can be precursors to later sexual behavior problems, and violence within the home can intensify this impact.

Research suggests that violence within the home, whether directed toward family members or toward the child, significantly increases the likelihood that sexual offending patterns will develop (Hunter \& Becker, 1994; Starzyk \& Marshall, 2003; Veneziano \& Veneziano, 2002). Witnessing abuse at home increases a child's likelihood of experiencing social, emotional, and behavioral problems during childhood and adolescence (Jaffe, Suderman, \& Reitzel, 1992). In fact, in a review of the literature, 
Jaffe, Suderman, and Reitzel (1992) found that boys who witness their mother's physical assault by a male (i.e., father or male partner) show consistent signs of externalizing as well as internalizing the events. Externalizing the abuse may take the form of fighting, destructive behavior, and forced sexual acts (Jaffe et al., 1992). Internalizing may be reflected in the development of emotional problems (Jaffe et al., 1993). Witnessing domestic violence at a young age has consistently been linked to adult and adolescent sexual offending (Gray, Busconi, Houchens, \& Pithers, 1997; Gray, Pithers, Busconi, \& Houchens, 1999). More than half (52\%) of the caregivers of adolescents with sexual behavior problems reported physically abusing his/her partner (Gray et al., 1999). Moreover, $87 \%$ of these adolescents reported witnessing the domestic abuse (Gray et al., 1999). These findings make it clear that witnessing domestic violence impacts a child negatively, but personally experiencing abuse may have more significant and long-term effects.

Parental or caregiver abuse toward the child has been associated with sexual offending in later years. It has been theorized that the experience of abuse at a young age, particularly for boys, fosters feelings of powerlessness, confusion, and a lack of control (Ryan, 1987). In order to compensate for these feelings, children and adolescents may respond with aggression and forced sexual behavior (Ryan, 1987). In fact, all types of childhood maltreatment, including sexual abuse, physical abuse, emotional abuse, and neglect, have been found to significantly predict sexual behavior problems that resemble sexual offending behavior in adults and adolescents (Gray et al., 1997; Gray et al., 1999; Hunter \& Becker, 1994; Starzyk \& Marshall, 2003; Veneziano \& Veneziano, 2002). 
Not surprisingly, the most common type of abuse perpetrated by a parent or caregiver that is associated with the development of sexual offending is sexual abuse (Barbaree \& Langton, 2006; Gray et al., 1997; Gray et al., 1999; Knight \& Prentky, 1993; Pithers \& Gray, 1998; Ryan, Lane, Davis, \& Isaac, 1986; Starzyk \& Marshall, 2003; Veneziano \& Veneziano, 2002). Early studies reported that as high as $81.25 \%$ of sexually aggressive adolescents have a reported history of sexual abuse (Friedrich \& Luecke, 1988; Johnson, 1988). In a meta-analysis of the literature on juvenile sex offenders in 1993, Kendall-Tackett, Williams, and Finkelhor found that $28.9 \%$ of juveniles with sexual behavior problems report having experienced CSA. More recently, literature has confirmed that sexual victimization serves as a significant predictive factor of sexual offending. In their study on juvenile sex offenders' self-esteem, Marshall and Mazzuco (1995) found that a large percentage (41.7\%) of juvenile sex offenders reported experiencing CSA as compared to a much smaller number of community controls $(8.7 \%)$. Other studies have found even higher rates of CSA in adolescents with sexual behavior problems. For instance, Gray et al. (1999) found that $84 \%$ of the adolescents who were referred to a treatment program for sexually inappropriate behavior reported having experienced CSA themselves. The rates of CSA in juvenile sex offenders and adolescents with sexual behavior problems are high, and sexual abuse is not the only form of child maltreatment that has been associated with later sexual offending. Physical abuse is the second most common form of child maltreatment that has been linked to adolescent sexual offending (Gray et al., 1997; Gray et al., 1999; Pithers \& Gray, 1998). An early investigation on prepubescent youth with sexual behavior 
problems indicated that $19 \%$ of cases involve a history of physical abuse (Johnson, 1988). Another study found that physical abuse during childhood was reported by juvenile sex offenders characterized as rapists at significantly higher rates than juvenile delinquents who committed non-sexual crimes (Knight \& Prentky, 1993). Physical abuse is often clearly recognized because children who experience it can have obvious physical symptoms. On the contrary, the rates other types of maltreatment, including emotional abuse and neglect, are considered underestimates due to the ambiguous nature of the symptoms that accompany these forms of maltreatment.

Like physical abuse, emotional abuse and neglect serve as significant predictive factors related to juvenile sexual offending (Gray et al., 1997; Gray et al., 1999; Pithers \& Gray, 1998). For example, Knight and Prentky (1993) found that juvenile sex offenders characterized as child molesters reported significantly more neglect by parents during childhood than did juvenile delinquents who committed non-sexual crimes. Similarly, Gray et al. (1997) found high rates of emotional abuse and neglect in adolescents with sexual behavior problems, $33 \%$ and $18 \%$, respectively.

Current literature also highlights the significant nature of simultaneously experiencing multiple forms of child maltreatment. Domestic abuses against children may co-occur and combinations between neglect and emotional, physical, and sexual abuse may further increase the likelihood of future sexual offending (Gray et al, 1999). In their research, Gray et al. (1997) found that $38 \%$ of juveniles with sexual behavior problems experienced both physical and sexual abuse as children. The deleterious effects of this combination of maltreatment are compounded by lack of resources and 
inappropriate coping models from emotionally abusive and neglectful parents (Barbaree \& Langton, 2006). These types of abuses against a child may also indicate an ongoing sexual offending pattern within the family.

Research has also uncovered the fact that sexual offending may be characteristic of some families in general. For example, Gray et al. (1999) found that $62 \%$ of extended families of an adolescent with sexual behavior problems had at least one other member who committed some form of sexual offending. Moreover, for families of adolescents with sexual behavior problems, Gray et al. (1997) found an average of 1.3 additional sex offenders, reported or unreported, in the family. In combination with experiencing and witnessing various forms of maltreatment, the presence of family members who commit sexual crimes, especially against members of their own family, only serves to perpetuate the cycle of violence and foster deviant sexual manifestations.

Since poor relationships with parents, maltreatment, and negative family experiences have been established as significant predictors of sexual offending against children, it is important to identify and examine the family dynamics that underlie adolescent sexual offending. In the discussion of the literature on family relationships and juvenile sexual offending thus far, however, an important qualification has been ignored, that of ethnic group differences. There are only a few empirical articles that investigate ethnic group differences and family dynamics in relation to juvenile sexual offending. Further distinguishing between these few research studies is the operational definition of family relationships. Family relationships have been measured through various scales assessing dimensions which include family cohesion, family conflict or hostile home 
environment, familism, monitoring, communication, and attitudes (Bischof, Stith, \& Wilson, 1992; Gorman-Smith, Tolan, \& Zelli, 1996; Meyerson et al., 2002; Miller, Forehand, \& Kotchick, 1999; Schechter, Brunelli, Cunningham, Brown, \& Baca, 2002; Sefarbi, 1990). Furthermore, reported differences in family relationships between ethnic groups appear to depend on the particular conceptualization of family relationships. For instance, some research on family cohesion, familism, and monitoring indicate that there are differences between juvenile offenders from different ethnic backgrounds (Bischof et al., 1992; Gorman-Smith et al., 1996). Other studies, operationally defining family relationships more broadly (e.g., communication and attitudes), suggest that there are no differences between ethnic group identity and family relationships in juvenile sexual offenders (Meyerson et al., 2002; Miller et al., 1999). On the surface, these findings seem contradictory; however, they completely depend on the definition of family relationships. In the present study, there is a focus the quality of parent (supervisor)-child relationships as reflected in communication, attitudes, and parent-child interactions.

Although some research suggests that juvenile sexual offenders from different ethnic backgrounds do not differ in family communication and attitudes, it is important to recognize that family structure and family values are by no means universal. Cultural experiences (i.e., acculturation) may have significant impacts on family dimensions like family dynamics, beliefs, and value; therefore, the influence of a larger cultural context must also be taken into account. More specifically, the impact of acculturation, or the process of individual and group cultural learning as a result of consistent contact between two or more cultures, must be assessed. Acculturation has been associated with family 
relationships in numerous studies (Baer \& Schmitz, 2007; Gil \& Vega, 1996; Miranda et al., 2000; Rodriguez, Mira, Paez, \& Myers, 2007; Romero, Robinson, Haydel, Mendoza, \& Killen, 2004; Rumbaut, 2001; Sabogal et al, 1987). Family relationships have been found to vary depending on acculturation level or strategy (Baer \& Schmitz, 2007;

Romero et al., 2004). Research has also investigated the impacts of family relationships and acculturation on psychological stress, environment, adaptability, and self-esteem (Gil \& Vega, 1996; Miranda et al., 2000; Rumbaut, 2001). Since acculturation has been found to interact with family relationships on a number of outcomes, a thorough understanding of acculturation and its impact on CSA is imperative.

\section{Acculturation}

The process of cultural learning whereby individuals or groups adapt or adopt one or more of a host culture's values, norms, beliefs and simultaneously maintain or reject the cultural heritage of one's country of origin is known as acculturation. Acculturation is a macrosocial, multidimensional construct in which continual contact between two or more cultures initiates the adaptation or adoption of one or more of the cultures (Berry, 2002; Berry, 2001; Marín \& Gamba, 2002). It is a bidirectional or multidirectional process resulting in cultural learning and change when multiple cultures come into consistent contact with one another (Berry, 2002; Trickett, 1996). Influence from both the dominant and the non-dominant cultures affect entire groups or individuals (Berry, 2002). The impact can be reactive as well as both direct or indirect and immediate or delayed (Berry, 2002). The two principle ways in which an individual's cultural identity 
can change reflect: (1) the "identification with one's heritage" and (2) the "identification with the large or dominant society" (p. 620; Berry, 2001). Further, identification with one's cultural heritage and identification with the dominant, host country culture are not mutually exclusive. Instead, cultural identification is contextually based on both continuums. In other words, individuals can make simultaneous changes on both dimensions (i.e., identification with one's heritage and identification with the dominant culture). Based upon these two dimensions, Berry $(2002 ; 2001)$ described four "strategies" of individual acculturation: (1) integration (combining elements from cultures of country of origin and host-country); (2) assimilation (disengagement from heritage and complete adoption of host culture); (3) separation or withdrawal (identify only with culture from country of origin); and (4) alienation or marginalization (complete withdrawal from traditions from country of origin as well as the alternate country; see Figure 1 for a multidimensional model of acculturation).

Some measures of acculturation have been criticized for their unidimensionality. Although Berry $(2002 ; 2001)$ suggests that acculturation is multidimensional, some researchers continually measure acculturation on a single continuum. For example, the Short Acculturation Scale and the Brief Acculturation Scale, developed by Marin, Sabogal, VanOss Marin, Otero-Sabogal, and Perez-Stable (1987) and Norris, Ford, and Bova (1996), respectively, are both unidimensional measures of acculturation where assimilation and separation are the end points of the continuum. Elements of these scales are present in multiple studies including those by Finch and Vega (2003), Samaniego and Gonzales (1990), Gil, Wagner, and Vega (2000), and Miranda, Estrada, and Firpo- 
Jimenez (2000). In unidimensional acculturation measures, it is assumed that Latinos who assimilate dismiss any and all ties to their heritage whereas Latinos who separate prohibit any integration of dominant cultural values with their own cultural values. Moreover, single continuum measures are problematic because they often dichotomize Latinos into two groups by level of acculturation (i.e., high and low). However, the acculturation process is much more complex than can be measured by a single continuum scale. Acculturation has an array of presentations (e.g., assimilation, integration, separation, marginalization) depending on the context of the situation (Birman, 1998; Coatsworth et al., 2005).

Multidimensional measures of acculturation do exist (Birman, 1998; Cuéllar et al. 1995; Marín \& Gamba, 1996; Phinney, 1992; Rodriguez et al., 2007). These measures typically include two distinct scales that indicate the individual's identification with each dimension of acculturation (i.e., culture of country of origin and culture of host country). One example of a multidimensional model of acculturation was created by Phinney (1992), called the Multigroup Ethnic Identity Measure and Other-group Orientation scale (MEIM and Other-group Orientation scale). This scale measures acculturation on two continuums: (1) ethnic group identification and (2) identification with other ethnic groups. Other variables, such as language usage, can be added to this scale for a more encompassing measurement of acculturation (Phinney, personal communication, October 30, 2009). However, most the research literature on acculturation does not use multidimensional model of acculturation in the conceptualization and measurement of acculturation. 
People of all origins can experience acculturation when they come into contact with a new or different culture. In the U.S., acculturative research has focused a great deal of attention on Latinos as a population of interest. Within Latino communities, research has shown variation between the four acculturative strategies (Coatsworth, Maldonado-Molina, Pantin \& Szapocznik, 2005; Cuéllar, Nyberg, Maldonado, \& Roberts, 1997). Cuéllar et al. (1997) found that young Latino adults who are more assimilated to American culture typically identified less with their heritage than those who maintained traditional values (separation) and those who integrated both cultures. Even though complete assimilation is encouraged in the U.S., Latino adolescents who are able to integrate both their own heritage and cultural values of the larger society show greater ability to adapt to psychosocial stressors (Coatsworth et al., 2005). Unfortunately, integration is the most difficult acculturative strategy because it involves the negotiation and navigation of two or more cultures (Berry, 2002; Taylor \& Lambert, 1996). Although integration has been found to be a successful strategy because individuals are able to adapt to various situations appropriately (e.g., at home, at school, at work), there is considerable heterogeneity in Latinos' methods of acculturation. The effects of different acculturative strategies vary, depending upon the person, situation, and environment.

The literature has examined the impact of acculturation on Latinos' physical and mental health as well as on health behaviors. Studies on physical health have demonstrated that acculturation can be detrimental. For example, one study found that highly acculturated (assimilated) Latinos provided self-reports of poorer physical health 
when compared with low acculturated Latinos (Finch \& Vega, 2003). In a meta-analysis of the literature on Latino health in relation to acculturation, Lara, Gamboa, Kahramanian, Morales, and Bautista (2005) found that studies consistently report that highly acculturated Latinos have poorer birth outcomes (e.g., prematurity, low birth weight, neonatal mortality) than less or non-acculturated Latinos. While these reports indicate that acculturation is negatively associated with physical health, studies on mental health show mixed results.

The literature on the mental health of Latinos is sparse, and studies have reported inconsistent findings. Some research suggests that both high acculturated Latinos with low ethnic identity and low acculturated Latinos with high ethnic identity have lower success and/or recovery rates once a mental illness is diagnosed (Gamst, Dana, DerKarabetian, Aragón, Arellano, \& Kramer, 2002). The meta-analysis conducted by Lara et al. (2005) states that research on mental health is, however, limited and inconsistent. Some of these inconsistencies may be a result of investigating varying illnesses, multiple definitions of illness, and different degrees to which individuals are affected. Some acculturative strategies may, in fact, be more beneficial to an individual depending on the particular situation and illness. It is difficult to determine specific trends across mental health because mental health depends on an appropriate person-situation match. There are, however, general trends in the literature on health behaviors worth noting.

With the exception of physical exercise, the literature consistently demonstrates that acculturation is positively associated with a variety of negative health behaviors. Illicit drug use, drinking (especially by women), smoking, poor nutrition, and poor 
behaviors during pregnancy, such as smoking and drinking, have all been linked Latinos' high acculturation (Lara et al., 2005). Although Lara et al. (2005) noted that some studies indicate conflicting results, the general trend for the impact of acculturation on healthcare coverage is positive. Consistent findings support the positive correlation between acculturation and the use of healthcare services, particularly preventive services (Lara et al., 2005). Despite the general trends found for health behaviors, appropriate conclusions can only be drawn when viewed within the context of cultural influences (e.g., acculturation).

Other behaviors, including criminal activity and delinquency, have only been studied minimally in relation to acculturation. In general, research suggests that highly acculturated Latinos are at greater risk for adolescent delinquency compared to Latinos that are low in acculturation (Fridrich \& Flannery, 1995). Studies that have investigated acculturation and delinquency bidimensionally have posited that assimilation and separation are associated with an increased risk of Latino adolescent delinquency whereas separation is related to a decreased likelihood of Latino delinquency (Berry, 2002; Buriel, Calzada, \& Vasquez, 1982; Vega, Gil, Warheit, Zimmerman, \& Apospori, 1993). There has been no research on the acculturation strategy known as integration in relation to adolescent sexual offending.

Acculturation and adolescent delinquency have also been linked to family relationships (Samaniego \& Gonzalez, 1999; Sullivan, Schwartz, Prado, Huang, Pantin, \& Szapocznik, 2007). For example, Sullivan, Schwartz, Prado, Huang, Pantin, and Szapocznik (2007) found that Latino adolescents characterized as assimilated reported 
lower levels of parental involvement, negative parenting, and less family support than adolescents characterized as integrated. The authors also found that assimilation was significantly related to Latino adolescent delinquency whereas integration and moderate acculturation were not (Sullivan et al., 2007). There is no research, however, on the associations between family relationships, adolescent delinquency, and other acculturation strategies such as separation and marginalization.

Although it has been established that acculturation and family relationships are essential to understanding all types of delinquency for diverse populations (Sullivan et al., 2007; Watts, 1992), studies on acculturation and family relationships have not extended into investigations on the perpetration of CSA by juvenile offenders. While there is evidence linking acculturation strategies to the delinquent behavior of some Latino adolescents (Fridrich, 1995), there are no studies that investigate the impact of acculturation on the perpetration of CSA. Research on acculturation and juvenile sex offending by Latino youth is needed to better understand factors that influence the perpetration of CSA. Since acculturation and family relationships have already been linked in other descriptions of criminal behavior (i.e., adolescent delinquency) by Latinos, the inclusion of acculturation as a construct would compliment and advance the current literature on family relationships and juvenile sexual offending. Furthermore, preliminary work done within the Latino community identified acculturation and family as important variables in relation to the perpetration of CSA; however, there have been no studies to date that examine the impact of these variables, in combination, on sexual offending, particularly in the juvenile sexual offender population. 


\section{Purpose of the Present Study}

As previously outlined, there is a lack of information on family relationships of perpetrators of CSA from minority populations, specifically from a Latino background. Family relationships, however, may change as Latinos navigate the acculturation process. Although there is evidence linking acculturation and adolescent delinquency, no studies have been conducted to examine the relationship between acculturation and juvenile sexual offending. Moreover, in relation to the literature on the perpetration of CSA, there is no empirical research that combines family relationships and acculturation.

The purpose of this study was to better understand the relationship between family relationships and the perpetration of CSA through the inclusion of acculturation strategies of Latino adolescents. Research questions, therefore, reflected the gaps in the literature in combining family relationships and acculturation in the study of juvenile sexual offending. The following section presents each research question and its corresponding hypothesis.

\section{Research Question 1}

The first research question assessed the relationship between acculturation and adolescent group membership. More specifically, can acculturation strategy predict group affiliation (i.e., juvenile comparison or juvenile sex offender)? High acculturation, or assimilation, has been associated with delinquent behavior as compared to low acculturation, or separation (Fridrich, 1995; Samaniego \& Gonzalez, 1999; Vega, 1993). 
Hypothesis 1.1: It was anticipated that adolescents characterized as assimilated or marginalized, as opposed to integrated or separated, are more likely to belong to the juvenile sex offender group.

Hypothesis 1.2: Adolescents characterized as integrated or separated, as compared to those assimilated or marginalized, are more likely to be affiliated with the juvenile comparison group.

\section{Research Question 2}

The second research question in the current study evaluated the relationship between acculturation and family relationships and their impact on adolescent group membership. Poor family relationship quality has been found to be a significant risk factor for adolescent sexual offending (Barbaree \& Langton, 2006; Starzyk \& Marshall, 2003; Veneziano \& Veneziano, 2002). Moreover, studies suggest that juvenile comparisons score higher on family relationship scales as compared to juvenile sex offenders (Bischof \& Stith, 1992; Bischof \& Stith, 1995). Research also indicates that acculturation serves as a mediator of the relationship between a family-related scale (i.e., familism) and juvenile group affiliation (e.g., delinquent vs. non-delinquent background; Schwartz et al., 2005). Measures within this study focus more on quality of relationship with a supervisor (family member supervisors only), rather than "family relationships" per se. Therefore, this study discusses supervisor relationship quality in lieu of family relationships. 
Hypothesis 2: It was hypothesized that acculturation mediates the relationship between supervisor relationship quality and juvenile group membership. Refer to Figure 2 for the mediated model.

\section{Methods}

\section{Participants}

The current study was part of a larger, ongoing investigation by Dr. Keith Kaufman and colleagues on the supervision and offending patterns (modus operandi) of juvenile sex offenders ${ }^{2}$. The original sample included 606 juvenile sexual offenders (JSOs) and juvenile comparisons (i.e., community adolescents with no criminal history; JCs) in five different states (Florida, Oregon, New York, South Carolina, and Texas). Of the original sample, 523 participants were included in this study. Data from JSOs was collected at juvenile offender facilities in each of the five states, and data from JCs was collected at community centers from each state. All participants were male and between the ages of 12 and 17 with a mean of 14.32 years (SD 1.54). This study compared four different, self-reported ethnic identities (i.e., African American, 19.5\% of the sample; European American, $46.7 \%$ of the sample; Latino, $19.9 \%$ of the sample; and Mixed ethnicity, $14.6 \%$ of the sample) in relation to the degree to which participants report high or low family relationship scores. Approximately 53 percent of the participants were affiliated with the JSO group and approximately 47 percent belonged to the JC group. Refer to Table 1 for a breakdown of participants' group affiliation and ethnicity. Approximately half (49.0\%) of all the participants resided in Oregon; however, the

\footnotetext{
${ }^{2}$ The larger study was funded by the Centers for Disease Control and Prevention (CDC Grant R49/CCR016517-01).
} 
majority $(55.8 \%)$ of data from Latino participants came from the State of New York.

Refer to Table 2 for the frequencies of participants from each ethnic group by state.

Because one of the primary purposes of this study is to take a population-specific approach to studying CSA, this study only examined Latinos for analyses concerning the hypotheses.

Design

This study utilized a cross-sectional, non-experimental design. Participants were asked to complete all questionnaires at the same time and were sampled only once during the course of the study. This study compared a group of juvenile sex offenders with a group of juveniles without any known criminal history (i.e., juvenile comparisons). Analyses primarily concentrated on participants that self-identified as Latino. Descriptions and Measurement of Study Constructs

Supervisor Relationship Quality (SRQ). To assess supervisor relationship quality, four questions in the Supervision Questionnaire (SQ; Kaufman, 2001) were utilized. This questionnaire was designed for the original, larger CDC study and included multiple subscales assessing perceived relationship with supervisor. These subscales will be used to evaluate family relationships and will be referred to as "supervisor relationship quality" (SRQ). The first scale identified an adolescent's primary caregivers during the year prior to his incarceration (SQ Part 1, Questions 3a-s; see Appendix A). Participants were provided with a list of 18 potential supervisors/caregivers (e.g., mother, father, grandmother, uncle, teacher, teenage baby-sitter) and were asked to mark the person(s) 
that supervised them during 4 time points (i.e., weekdays during the school year, evenings during the school year, weekdays during the summer, and weekends and school vacations). There were 13 potential family members within the list of caregivers, and only those participants that indicated that at least one of the 13 caregivers provided them supervision were included in analyses.

Subscales within the SQ specifically pertaining to supervisor relationship quality contained behavioral and attitudinal elements. For example, SQ Part 1, Questions 30a-g (see Appendix B) asked participants how often the adolescent and his caregiver did various activities together. These items were measured on a 5-point Likert scale ranging from 0 (never) to 4 (always). Example items from this subscale include: "My supervisor and I did activities together (like played games)" and "My supervisor helped me with my homework." SQ Part 1, Questions 32a-n (see Appendix C) also measured behavioral elements of family relationships by asking participants how often they discussed specific topics with their caregiver. These items were also measured on the same 5-point Likert scale ranging from 0 to 4 . Examples of items within this subscale include: "How often did you talk to your supervisor about your school work?" and "How often did you talk to your supervisor about your friends?"

Finally, items within another subscale (SQ Part 1, Question 31; see Appendix D) of the SQ evaluate attitudinal elements of supervisor relationship quality. These items are also measured on the same 5-point Likert scale from 0 to 4 . Examples of these attitudinal supervisor relationship quality items include: "My supervisor accepted me for who I was." and "My supervisor understood where I was coming from." 
Acculturation. To measure acculturation, the current study utilized the Multigroup Ethnic Identity Measure (MEIM) and the Other-group Orientation Scale developed by Phinney (1992; see Appendix E for MEIM + Other-group Orientation Scale). The MEIM consists of three subscales: (1) affirmation and belonging; (2) ethnic identity achievement; and (3) ethnic behaviors. Alone, the MEIM only evaluates an individual's identification toward the culture from his/her country of origin. With the incorporation of the Other-group Orientation scale, identification with other ethnic groups was also measured. The MEIM was comprised of 14 items, and the Other-group Orientation scale included 6 items. The MEIM and Other-group Orientation scale was measured on a 4point Likert scale ranging from 1 (strongly agree) to 4 (strongly disagree). The combination of these two scales measured ethnic identity on two dimensions. To supplement the information gained from the MEIM and Other-group Orientation Scale, another scale within the Demographics Questionnaire pertaining to Language Usage was utilized (see Appendix F for Language Usage Scales). The Language Usage scales identified language usage (i.e., Spanish, English, or Other) during various activities and were measured on a 5-point Likert scale from 0 (never) to 4 (always). For the purposes of this study, only the language information for Spanish and English was utilized. The combination of the MEIM and Other-group Orientation Scale and Language Usage Subscales allowed for the categorization of acculturation into each of the four acculturation strategies.

Procedures 
Juvenile sex offender (JSO) participants were recruited from juvenile detention facilities in 5 different states (i.e., FL, OR, NY, SC, and TX). Juvenile comparisons (JCs) were recruited from various communities within the same states. For JSOs, representatives of the state facilities who had custody of these adolescents provided consent for participation. Participants in state facilities were also provided with an assent form, which was read aloud to them. Consent for JC participation was provided by a parent or guardian, and JC participants were also given an assent form to complete. Participation was voluntary, and all responses were kept confidential. All participants were also screened for reading level, comprehension abilities, and significant mental disabilities. Once participants were screened and consented to take part in the study, they were given three questionnaires which included the Demographic Questionnaire (part of which is the acculturation scale, the MEIM; see Appendix E; Kaufman, 2001) and the Supervision Questionnaire (SQ; see Appendices B, C, and D; Kaufman, 2001). Participants also completed a measure designed to assess their patterns of perpetration, the Modus Operandi Questionnaire (Kaufman, 1994). Findings from this measure were not included in this study. It typically took between 45 and 60 minutes for participants to complete the Demographic Questionnaire and the SQ. Once the questionnaire packets were completed, they were handed to a research assistant and taken to Portland State University where they remain triple-locked. 
Results

\section{Participants}

Before analyses were conducted, exclusion criteria were applied to the sample population. Female participants or those that did not indicate their sex $(n=17)$ were not included in analyses. Participants indicating that their age, prior to incarceration, was less than 12 or greater than 17 years or did not indicate their age prior to incarceration (n $=62$ ) were excluded from the study. The age of the participant was calculated for the year prior to his incarceration because participants were asked to complete the SQ for the year prior to his incarceration. Participants were asked to do so in order to better understand their relationship with their supervisors before they were arrested for sexual offending. Participants that reported that they were not supervised by any family member (i.e., court supervised or self-supervised; $n=3$ ) were also excluded from analyses. Finally, one participant was excluded from data analyses for what appeared to be patterned responses.

The sample size varied for each analysis (see Table 3). For descriptive and initial inferential analyses, sample sizes were large, with a sample size of 523 for analyses using the whole sample and a sample size of 104 for analyses pertaining to the Latino subsample. However, the sample size dropped significantly for exploratory factor analyses because the statistical program utilized (i.e., SPSS 17.0) conducts an EFA using listwise exclusion. Sample sizes for CFAs were based on the entire sample or subsample because the statistical program (i.e., AMOS 7.0) uses a maximum likelihood technique that is able to estimate responses if not already provided. Since the cluster analysis 
depended on complete responses, sample size $(n=46)$ was also small. However, the sample size $(\mathrm{n}=93)$ was significantly improved when results from median splits were utilized instead of results from the cluster analysis. Since samples sizes varied between analyses, the sample size will be clearly stated in the description of each analysis mentioned above in the following sections.

All participants were between the ages of 12 and $17(M=14.32, S D=1.54)$. Refer to Table 4 for a breakdown of age (ethnicity X group). A 2 X 4 Factorial ANOVA was conducted on all 523 participants to examine age differences between groups and participants of different ethnic backgrounds. The main effect for group was significant, $\mathrm{F}(1,515)=20.04, p<.05$, partial $\eta^{2}=.04$, indicating that the JC group was significantly older $(M=14.69, S D=.11)$ than the JSO group $(M=13.99, S D=.11)$. There were no significant age differences between the 4 ethnic groups, $\mathrm{F}(3,515)=0.45, p=.71$, partial $\eta^{2}=.00$, and the interaction between group and ethnicity was also found to be nonsignificant, $\mathrm{F}(3,515)=2.01, p=.11$, partial $\eta^{2}=.01$.

An independent samples $t$-test was conducted to examine age differences between groups in the Latino subsample $(\mathrm{n}=104)$. Results did not reflect the ANOVA findings on the whole sample. Instead, JCs were not significantly older $(M=14.41, S D=1.73)$ than JSOs $(M=14.12, S D=1.34)$ in the Latino subsample, where equal variances were not assumed, $t(79)=-.92, p=3.6, \mathrm{~d}=-.20$. Potential age differences, whether significant or not, did not pose a threat to further analyses because the maximum age difference between groups was only approximately 7 months and between Latino participants the 
age difference was only about 4 months. Furthermore, all participants were within the same developmental time period (Dahl, 2004).

To better understand the educational background of participants and to compare educational backgrounds between groups, the educational achievement of all participants was also explored. A 2 X 4 Factorial ANOVA was conducted on 461 participants to investigate educational attainment (i.e., grade completion) differences between groups and ethnicities. Both the main effect for group and the main effect for ethnicity, $\mathrm{F}(1$, $453)=17.99, p<.05$, partial $\eta^{2}=.04$ and $\mathrm{F}(3,453)=3.38, p<.05$, partial $\eta^{2}=.02$, respectively, were found to be statistically significant. These findings, however, must be considered together in light of the statistically significant interaction, $\mathrm{F}(3,453)=4.91, p$ $<.05$, partial $\eta^{2}=.03$. Although JSOs completed more education $\left(M=10^{\text {th }}\right.$ grade, $S D=$ .13 grades $)$ than JCs $\left(M=9^{\text {th }}\right.$ grade, $S D=.13$ grades $)$, the significant interaction suggested that the most disparate educational levels were between the EuropeanAmerican subsamples (see Figure 3). European-American JSOs had the highest educational attainment of any ethnic group, almost reaching the $11^{\text {th }}$ grade, and European-American JCs had the lowest educational completion, just beginning the $9^{\text {th }}$ grade.

An independent samples $t$-test was also conducted to confirm these results in the Latino subsample $(n=86)$. Findings reflected those found in the $2 \mathrm{X} 4$ Factorial ANOVA, where Latino JSOs completed more education $\left(M=10^{\text {th }}\right.$ grade, $S D=1.59$ grades) than Latino JCs $\left(M=9^{\text {th }}\right.$ grade, $S D=1.84$ grades $)$, where equal variances assumed, $t(84)=2.10, p<.05, \mathrm{~d}=.56$. These differences may seem surprising 
considering that JCs were significantly older than JSOs; however, JSO participants' age was calculated for the year prior to incarceration where as grade completion was reported for time of measurement. Age was based on the year prior to incarceration because the researcher wanted to know the age of the participant for the year in which he was reporting on his relationship with his supervisor (i.e., the year prior to incarceration). There were differences in the number of years between incarceration and time of measurement for JSOs. Furthermore, these differences helped to explain why JSOs, although younger for the year prior to incarceration, had higher grade completion than the JC group.

Prior to conducting inferential analyses on supervisor relationship quality, data on primary supervisor/caregiver was examined. In order to characterize the primary supervisor for each ethnic group (i.e., African Americans, European Americans, Latinos, and Mixed) as well as for each juvenile group (i.e., JC and JSO), frequency statistics were calculated. From these statistics, the top three supervisors were identified by the percentage of participants who identified each family member as his supervisor (see Table 5). African American, European Americans, and participants that identified as Mixed ethnicity all reported that their top three family supervisors were, in order of primary supervision, the mother, the father, and the grandmother. The top three supervisors for these ethnic groups were the same for both the JSO and JC groups. Latinos reported the mother, the father, and the aunt (in order of primary supervision) as the top three supervisors. Again, the top three supervisors were the same for both the Latino JSO and JC groups. 
From the preliminary, descriptive results, the mother, the father, and the aunt were the top three supervisors reported by Latino participants. However, the percentage of participants reporting supervision between these familial supervisors was seemingly different. To further explore these differences in the Latino subsample, three chi-square tests for independence were conducted. Only the chi-square test for independence examining the differences in reporting the mother as the primary supervisor was significant, $\chi^{2}(1, N=104)=14.86, p<.05, \Phi=.38$, indicating that JCs reported being supervised by their mother significantly more than JSOs, even though the mother was the primary caregiver for both groups. Results from the chi-square tests for independence for both the father (as the second most reported supervisor) and the aunt (as the third most reported supervisor) were non-significant, $\chi^{2}(1, N=104)=1.30, p=.25, \Phi=.11$ and $\chi^{2}(1, N=104)=.01, p=.92, \Phi=.01$, respectively, indicating that JCs were no more or less likely to report being supervised by their father and aunt than JSOs.

A 2 X 4 Factorial ANOVA was conducted on all 523 participants to identify any significant differences in the number of family supervisors for each adolescent group and ethnicity. Both of the main effects, testing differences in the number of family supervisors per adolescent group and per ethnic group, were found to be non-significant, $\mathrm{F}(1,515)=.33, p=.57$, partial $\eta^{2}=.00$ and $\mathrm{F}(3,515)=2.01, p=.11$, partial $\eta^{2}=.01$, respectively. The interaction between adolescent group affiliation and ethnic background was also found to be non-significant, $\mathrm{F}(3,515)=.03, p=.99$, partial $\eta^{2}=.00$. These findings suggest that there were no significant differences in the mean number of family 
supervisors between the two adolescent groups (JCs and JSOs) and the four ethnic groups (African-American, European-American, Latino, and Mixed ethnicity).

To inform subsequent analyses concerning the hypotheses, which were conducted exclusively on the Latino subsample, an independent samples $t$-test was also conducted to examine the mean number of family supervisors between juvenile groups in the Latino subsample $(n=104)$. Findings reflect those found in the 2 X 4 ANOVA, suggesting that there is no significant difference in the number of family supervisors between the JSO and JC groups within the Latino subsample, where equal variances assumed, $t(102)=.32$, $p=.75, \mathrm{~d}=.07$.

\section{Supervisor Relationship Quality: Structural Validity and Internal Reliability}

Questions pertaining to supervisor relationship quality were continuous Likert scale items, which range from 0 (never) to 4 (always). Because these scales had not been used with this population, specifically within the Latino subsample, structural validity and internal reliability (i.e., internal consistency) were addressed prior to analyses. To evaluate the structural validity, an exploratory factor analysis (EFA) was conducted on the three subscales for supervisor relationship quality. Three hundred and seventy-two participants from each of the four ethnic groups were included in the EFA on supervisor relationship quality. Twenty-seven items from the three subscales were entered into an EFA, using maximum likelihood (ML) and an oblique rotation for maximum factor fit. In the first round of analysis, one item (32h; "How often did you talk with your supervisor about: ...family issues?") was not salient (i.e., based on a criteria of a factor 
loading greater than or equal to .3; McDonald, 1999) on any factor, so this item was removed and the EFA was rerun. The second round of analysis indicated five factors for the 26 items within the three subscales. Factors were chosen based on the more conservative method of factor extraction, using eigenvalues of 1.0 or greater. Residual correlations between items satisfied cutoff criteria, all below |.1| (McDonald, 1999). Four items were salient on two factors, and these items were categorized under the most appropriate factor based on each item's content and the magnitude of the factor loading. Based on item content, each factor was named. Factor 1 was named "Daily Communication" as items pertained to discussion surrounding daily issues (e.g., "How often did you talk with your supervisor about: ...your school work?” and “...chores?”). Items on Factor 2 asked a participant about his perception of supervisor-participant relationship (e.g., "My supervisor trusted me" and "My supervisor accepted me for who I am") and were, therefore, named "Attitudes" toward relationship with supervisor. Factor 3 was named "Personal Communication" because item content referred to discussion about personal topics with the supervisor (e.g., "How often did you talk with your supervisor about: ...questions about sex?" and "...drugs and alcohol?"). Factor 4 was named "Activities" because these items asked about activities that the supervisor and participant did together (e.g., "My supervisor taught me things (like how to cook)" and "We went to the park together"). Finally, items loading on to Factor 5 dealt with "General Communication" about life (e.g., "How often did you talk with your supervisor about: ...something good that happened?" and “...your life?”). 
A third EFA was then conducted to introduce a higher-order factor (i.e., Supervisor Relationship Quality). Items loading onto each of the five factors were averaged (i.e., mean) to create a composite, relative test score for each factor. These composite scores were entered into an EFA as items. The higher order latent factor, Supervisor Relationship Quality, was introduced to predict these five composites. Factors were extracted based on eigenvalues greater or equal to 1.0. Only one factor was extracted, and a scree plot confirmed these results. A reproduced correlation matrix indicated that all residual composite item correlations were below the cutoff criteria of |.1|, between -.06 and .06 (McDonald, 1999).

To verify the factor structure obtained through the first- and second-order EFAs, a confirmatory factor analysis (CFA) was conducted. Refer to Figure 4 for the Supervisor Relationship Quality factor model. The CFA on the supervisor relationship quality factor structure included all 523 participants. Various constraints were placed onto the model before it was run. All error variance and disturbance loadings were constrained to 1, and one item factor loading on each of the first-order factors was also constrained to 1 (Keith, Fine, Taub, Reynolds, \& Kranzler, 2006). All items loaded significantly onto the five first-order factors, and all five of the first-order factors significantly loaded on the higher order factor. The chi-square goodness-of-fit index was significant, $\chi^{2}(294)=968.15, p<$ .05. This was not surprising as the $\chi^{2}$-statistic is especially sensitive to sample size (Wegener \& Fabrigar, 2000), in this case $N=523$. Due to its sensitivity to sample size, other model fit indices that evaluate incremental and absolute fit were calculated to further examine the factor model (Hu \& Bentler, 1999). The Comparative Fit Index 
(CFI), which is one example of an incremental fit index, suggested poor model fit (.87) as it was below .90 for adequate fit and well below .95 for good fit (Hu \& Bentler, 1999). However, the Root Mean Square Error Approximation (absolute fit index; RMSEA) indicated acceptable model fit (.07) as it was less than the .08 cutoff for adequate fit $(\mathrm{Hu}$ \& Bentler, 1999).

Factor analyses were also conducted on the Latino subsample to verify that the factor structure for Supervisor Relationship Quality held for Latinos. The Latino subsample size was small ( $\mathrm{n}=68$ for complete cases), so the EFA was not conducted in the same manner as the original, whole sample EFA. Instead, each factor from the original EFA was tested separately within the Latino subsample. Again, ML and oblique rotation techniques were utilized. All items from each factor loaded saliently (i.e., factor loading of .3 or greater; McDonald, 1999) onto each specified factor, and each EFA revealed only 1 factor. The higher order EFA reflected the results of the higher order analysis on Supervisor Relationship Quality in the larger sample. By extracting factors based on eigenvalues equal to or greater than 1.0 and examining a scree plot, only one second-order factor was found (i.e., Supervisor Relationship Quality) for the Latino subsample.

A CFA on Supervisor Relationship Quality, equivalent to that conducted on the whole sample population (see Figure 4), was conducted on the Latino subsample (n = 104). Although all items significantly loaded onto each of the 5 first-order factors and these first-order factors significantly loaded onto the second-order factor, the model was found to poorly fit the data. The chi-square goodness-of-fit test was significant, $\chi^{2}(294)$ 
$=543.67, p<.05$, and both the incremental and absolute fit indices supported the results of the chi-square analysis. The CFI (.73) was below the .90 cutoff for adequate model fit, and the RMSEA (.09) was above the .08 cutoff (Hu \& Bentler, 1999). Poor model fit was not surprising since the fit indices available (i.e., CFI and RMSEA) through the statistical package employed (AMOS 7.0) have been found to over-reject model fit for sample sizes less than 250 (Hu \& Bentler, 1999).

Attempts to improve the Supervisor Relationship Quality factor model were made by examining model fit for each of the first-order factors in the Latino subsample. All three first-order communication factors (i.e., General Communication, Daily Communication, and Personal Communication) were found to fit the data well. All items significantly loaded onto the General and Personal Communication factors. One item (32i) on the Daily Communication factor did not have a significant factor loading and was deleted. Then the model was rerun, and all items loaded significantly onto the Daily Communication factor. The chi-square goodness-of-fit analysis indicated good model fit for General Communication, Daily Communication, and Personal Communication models, suggesting that they were not statistically different from the saturated models, $\chi^{2}(2)=2.59, p=.27, \chi^{2}(5)=9.06, p=.11, \chi^{2}(2)=2.19, p=.33$, respectively. Since small sample sizes are more likely to produce non-significant chi-square results (Wegener \& Fabrigar, 2000), CFIs and RMSEAs were examined for each of the Communication first-order factors. All the CFIs (1.0, .94, and 1.0, respectively) and two of the three RMSEAs $(.05, .09$, and .03 , respectively) for each factor supported the chisquare test of independence results suggesting good model fit. 
CFAs were also conducted on the Attitudes and Activities factors. Results for the Attitudes factor were similar to the results on the communication factor analyses. All items significantly loaded on the Attitudes factor. Although the chi-square goodness-offit test was significant, $\chi^{2}(5)=12.77, p<.05$ and the RMSEA (.12) suggested poor model fit, the CFI (.96) indicated good model fit, above .95 (Hu \& Bentler, 1999). However, results from the CFA on the Activities factor supported the conclusion that the model was poorly fit to the data. Despite significant factor loadings, all fit indices suggested poor fit. The chi-square goodness-of-fit test was significant, $\chi^{2}(14)=30.94, p$ $<.05$, and the incremental $(\mathrm{CFI}=.88)$ and absolute $(\mathrm{RMSEA}=.11)$ fit indices did not match or better the cutoff criteria.

Using results from the CFAs on each of the five factors for Supervisor Relationship Quality, the larger, hierarchical factor model was fit a second time. This time, item $32 \mathrm{i}$ from the Daily Communication factor was deleted, and the entire Activities factor, including its items, was omitted from the model (see Figure 5). All items loaded significantly onto their appropriate first-order factor, and each of the five first-order factors significantly loaded onto the second-order, Supervisor Relationship Quality factor. However, all the fit indices still suggested poor model fit. The chi-square goodness-of-fit test was significant, $\chi^{2}(131)=277.42, p<.05$. The CFI (.78) was below the .90 criteria, and the RMSEA (.10) was equivalent to the cutoff score for poor model fit. Although the model fit was significantly improved, $\chi^{2}(163)=266.25, p<.05$, the second model still fit the data poorly. 
Since the Supervisor Relationship Quality factor structure had acceptable model fit for the whole sample population, one continuous Supervisor Relationship Quality score was calculated. Item responses on each of the five factors were averaged (i.e., mean), producing a score ranging from 0 to 4 . These five averages were then aggregated to form the second-order, Supervisor Relationship Quality score. The supervisor relationship quality scale ranged from 0 to 20 . This composite score was used to examine mean differences in the supervisor relationship quality between ethnic groups. For analyses concerning Latinos only (i.e., tests of Hypotheses 1 and 2), only the composite relative test scores for each of the 5 indicators (i.e., factors) of supervisor relationship quality were utilized.

Finally, the internal reliability (i.e., internal consistency) of each factor as well as the higher-order factor was evaluated using Cronbach's alpha ( $\alpha$; see Table 6$)$. All scales for the whole sample population as well as the Latino subsample had acceptable reliabilities $\alpha$ above .72 , and many scales had good reliabilities $\alpha$ above .80 (John \& Benet-Martínez, 2000).

In order to examine adolescent group affiliation and ethnic group differences in supervisor relationship quality scores, a 2 X 4 Factorial ANOVA was conducted on all 523 participants. Prior to analysis, four outliers were identified on the Supervisor Relationship Quality variable, all reporting low Supervisor Relationship Quality. Three of the four outliers belonged to the JSO group. After examination of the four cases, it was decided to include the outliers in the analysis as they are valuable sources of varying information. Results show that the main effect for adolescent group affiliation was 
significant, $\mathrm{F}(1,503)=8.36, p<.05$, partial $\eta^{2}=.02$. These findings indicate that, on average, the JC group reported significantly higher Supervisor Relationship Quality scores $(M=12.56, S D=.26)$ than the JSO group $(M=11.52, S D=.25)$. There were no significant differences in mean reported Supervisor Relationship Quality scores among the different ethnic groups, $\mathrm{F}(3,503)=2.18, p=.09$, partial $\eta^{2}=.01$, and there was no significant interaction in mean Supervisor Relationship Quality scores between adolescent group affiliation and ethnicity, $\mathrm{F}(3,503)=1.74, p=.16$, partial $\eta^{2}=.01$.

\section{Acculturation: Structural Validity and Internal Reliability}

Before categorizing participants into four acculturation strategies, structural validity and internal reliability were examined because the validity and reliability for the items pertaining to acculturation (i.e., items from the MEIM and Other-group Orientation scale and the Language Usage scales) had not been evaluated with this dataset. The subsample size of Latinos with complete data $(\mathrm{n}=31)$ was too small to run an EFA on all acculturation variables (i.e., 44 items on MEIM and Other-group Orientation, Spanish Language Usage, and English Language Usage). Therefore, three separate EFAs were conducted on each of the three subscales. Sample sizes for EFAs on the language usage scale were based on only those Latino participants with complete data (English Language Usage, $n=63$; Spanish Language Usage, $n=45$ ). A ML technique and oblique rotation were utilized for all EFAs. EFAs on both the English and Spanish Language Usage scales indicated a single-factor structure. Both methods to determine factor structure (i.e., eigenvalues of 1.0 or greater and scree plot) of the English Language Usage scale 
produced a single-factor structure. All English language items loaded saliently onto the single factor, named English Language Usage. Additionally, all residual correlations were within the appropriate bounds, between -.1 and .1 (McDonald, 1999). Although the two methods determining factor structure on the Spanish language items produced different results, a single-factor structure was chosen based on the scree plot indicating one factor. Results from the scree plot, as opposed to eigenvalues equal to or greater than 1.0, were used to determine factor structure to match the factor structure of the English Language Usage scale and because the eigenvalue method oftentimes overestimates the number of factors within a model (McDonald, 1999). All items loaded onto the Spanish Language Usage factor saliently and residual correlations were within the appropriate bounds.

Two separate CFAs were conducted on the Language Usage scales. Each of these CFAs included 104 Latino participants. All but one item loaded significantly onto the English Usage factor, and the factor structure was found to have adequate model fit. Refer to Figure 6 the English Language Usage factor model. The chi-square goodnessof-fit test was significant, $\chi^{2}(54)=82.27, p<.05$; however, significance was anticipated because the $\chi^{2}$ statistic is particularly sensitive to sample size (Wegener \& Fabrigar, 2000). Two other fit indices were evaluated to determine model fit. Although the CFI (.79) suggested poor model fit, the RMSEA (.07) suggested acceptable model fit as it was below .08 (Wegener \& Fabrigar, 2000).

A CFA on the Spanish Language Usage factor model was also conducted. Refer to Figure 7 for the Spanish Language Usage factor model. Although all items 
significantly loaded onto the Spanish Language Usage factor, results from the model fit indices did not indicate good model fit. Similar to the English Language Usage factor, the chi-square goodness-of-fit analysis was significant, $\chi^{2}(54)=138.96, p<.05$, for the Spanish Language Usage factor. Since chi-square significance was anticipated due to sample size, results from the CFI and RMSEA were also examined. Both fit indices indicated poor model fit for the Spanish Language Usage factor (CFI $=.73$ and RMSEA $=.12$ ). While the CFI should have been greater than .90, the RMSEA should have been below .10 for acceptable model fit (Wegener \& Fabrigar, 2000).

Before an EFA on the MEIM and Other-group Orientation scale was undertaken, four items (B-13, B-14, B-16, and B-21) were reverse coded, accordingly to Phinney's (1992) guidelines. Thirty-one participants were included in the EFA on the MEIM and Other-group Orientation scale. All 20 items of the MEIM and Other-group Orientation scale were entered into an EFA. Again, a ML technique and oblique rotation was conducted. In the first round of analyses, five factors were selected; however, one factor contained only one salient item (B-16). Therefore, this item was dropped. Six items (B12, B-13, B-14, B-19, B-20, and B-24) loaded saliently on to two factors, and 1 item (B26) saliently loaded on to three factors. The item that loaded onto three factors was dropped from analyses given the goal of creating interpretable factors. The EFA was then rerun. The second round of analyses produced similar results with five factors, one of which contained only one salient item (B-11). This item was dropped, and the EFA was run a third time. Both the factor extraction methods (i.e., eigenvalues equal to or greater than 1.0 and scree plot) for third EFA suggested a four-factor model. All residual 
correlations were within the acceptable bounds, less than $|.1|$ (McDonald, 1999). There were two complex items (B-17 and B-25), loading saliently onto two factors. Based on item content, these factors were assigned to the most appropriate factor (B-17 to Factor 1 and B-25 to Factor 3). One item (B-12) loaded saliently (.33) onto Factor 3; however, the item content did not match that of Factor 3 (relating to other-group orientation). This item was near salient (.29) on Factor 2, and its item content related better to Factor 2 (ethnic group clarity and contentment) than to Factor 3. Therefore, this item was moved to Factor 2. Based on item content, these factors were titled: "Belonging" for Factor 1 (e.g., "I have a strong sense of belonging to my own ethnic group"), "Ethnic Group Clarity" for Factor 2 (e.g., "I have a clear sense of my ethnic background and what it means for me"), "Other-Group" for Factor 3 (e.g., "I am involved in activities with people from other ethnic groups"), and "Active" in own ethnic group for Factor 4 (e.g., "I have spent time trying to find out more about my own ethnic group, such as its history, traditions, and customs"). Phinney (1992) found only two factors through an EFA, Ethnic Identity and Other-group Orientation. Her results combined the three factors found in this study relating to ethnic identity. All six of Phinney's Other-group Orientation items loaded saliently onto one factor (Factor 3 - Other-Group) in this study as they did in her 1992 study; however, the remaining items did not break out into the three categories (i.e., Affirmation and Belonging, Identity Achievement, and Behaviors) comprising her second factor. Finally, slight to moderate correlations were found between some of the factors (see Table 7 for a factor correlation matrix). 
Following the three EFAs on the MEIM and Other-group Orientation scale, a CFA $(n=104)$ was conducted on the four factors. As indicated in the EFA, the 4 correlations between Belonging and Clarity, Belonging and Active, Belonging and Other, and Clarity and Other were represented within the CFA. As expected, the chi-square goodness-of-fit test was statistically significant, $\chi^{2}(115)=174.24, p<.05$. Therefore, other model fit indices were inspected. The CFI (.85) was nearly adequate, approaching .90 , and the RMSEA (.07) was considered reasonable, between .05 and .08 (Wegener \& Fabrigar, 2000). Overall, results from the CFA indicated sufficient model fit. Refer to Figure 8 for the MEIM and Other-group Orientation factor model.

Cross-validation of facture structure and model fit was not conducted on any of the scales due to the small sample size $(\mathrm{N}=104$; for the complete Latino data: $\mathrm{n}=31$ for the MEIM and Other-group Orientation scale, $n=63$ for English Language Usage, and $n$ $=45$ for Spanish Language Usage). Following factor analyses, a relative test score was calculated for each of the six factors based on the items that loaded onto each of these dimensions. Relative test scores were based on pairwise exclusion, using an $80 \%$ response rate or higher for each set of items. The relative test scores based on the four factors from the MEIM and Other-group Orientation scale ranged from 1 to 4 . Higher scores on the Belonging, Ethnic Group Clarity, and Active factors indicated higher levels of ethnic group identification for each factor, and a higher score on the Other-Group factor suggested higher Other-group orientation. Relative test scores on the Language Usage scales ranged from 0 to 4, where higher scores on each factor indicated more use of the specified language. These scores were used to determine acculturation strategy. 
To evaluate internal reliability, Cronbach's alpha $(\alpha)$ was calculated on the 4 MEIM and Other-group Orientation scale factors as well as the two Language Usage factors. Refer to Table 6 for scale reliabilities. Two internal reliability (i.e., internal consistency) scores failed to reach acceptable alpha levels (i.e., Ethnic Group Clarity and Active) most likely because each of these factors consists of only three items. It should be noted that the Active factor (Cronbach's $\alpha=.66$ ) approached a satisfactory alpha level, only .06 units short of the .72 cutoff (John \& Benet-Martínez, 2000).

\section{Acculturation Strategies}

Before a cluster analysis was conducted, relationships between each of the six acculturation factors (i.e., English Language Usage, Spanish Language Usage, Belonging, Ethnic Group Clarity, Active, and Other-Group) were examined. Refer to Table 8 for a correlation matrix. Not surprisingly, the Belonging, Ethnic Group Clarity, and Active factors were slightly to highly correlated in a positive direction. It is also not surprising that the correlation between English and Spanish Language Usage, although small, was negative. The Other-group factor had a positive, slight correlation with English Language Usage and a slight, negative correlation with Spanish Language Usage. It may, however, be problematic that the Other-Group factor was highly correlated with Belonging and Ethnic Group Clarity (.50 and .48, respectively).

Using the six factors for acculturation as the input variables, a hierarchical cluster analysis was conducted to categorize Latino participants $(\mathrm{N}=104)$ into different acculturation strategies. Squared Euclidean Distance was used to measure group distance, 
and Ward's method was utilized to cluster the groups. These methods have both been found useful in the Community Psychology literature (Rapkin \& Luke, 1993). From the dendogram (see Figure 9) in this initial analysis, three clusters were identified. Participants within the first cluster $(n=20)$ were categorized as Separated. Participants in the second cluster $(n=11)$ were categorized as Assimilated, and participants in the third cluster $(\mathrm{n}=10)$ were categorized as Integrated.

To validate the cluster structure, multiple analyses were conducted. The first validation method conducted was a series of ANOVAs to examine cluster differences on the variables used for cluster specification for the 31 participants associated with the clusters. Refer to Table 9 for the means and standard deviations of each group and Table 10 for post hoc ANOVA results for the mean differences in cluster variables between cluster groups. Post hoc comparisons indicated that the Assimilated cluster reported significantly less use of the Spanish language $(M=1.31, S D=.17)$ than the Separated $(M$ $=2.33, S D=.13)$ and Integrated $(M=2.76, S D=.18)$ clusters. Post hoc analyses also revealed that the Separated cluster reported significantly less English usage $(M=2.71$, $S D=.10)$ compared to the Assimilated $(M=3.38, S D=.14)$ and Integrated $(M=3.61$, $S D=.15)$ clusters. The most important finding, however, was that there were no significant mean differences between the three clusters on Other-Group variable, and all three clusters reported a relatively low (all means below 2.0) other-group orientation. This suggested that even the participants within the Assimilated cluster may not have identified with another ethnic group as much as they did their own ethnic group. 
Although the first validation method was flawed because there were no significant differences in the identification with other ethnic groups, two 3 X 3 chi-square analyses were conducted to assess the validity of the cluster structure using variables that were not utilized in the cluster analysis. The first chi-square analysis evaluated cluster differences on a different measure of language usage (i.e., "What languages do you speak fluently...Spanish, English, Other”). There were no significant differences in language usage (speaking fluently) between the 3 clusters, $\chi^{2}(6, N=41)=3.26, p=.78$, Cramer's $\mathrm{V}=.20$. The second chi-square analysis examined the ability to write fluently (i.e., "What languages do you write fluently...Spanish, English, Other") between clusters and found similar results. There were no differences in ability to write fluently in any language between the 3 clusters, $\chi^{2}(8, N=41)=7.04, p=.53$, Cramer's $\mathrm{V}=.29$. These findings suggested that the cluster structure was not valid.

Two final attempts were made to validate the cluster structure. The first attempt examined the cluster structure of the data using different agglomeration techniques. None of the hierarchical cluster analyses using Squared Euclidean Distance - Centroid techniques, Squared Euclidean Distance - Between-groups techniques, Correlation Between-groups techniques, and Correlation - Within-groups techniques resulted in similar cluster structures. Finally, a K-means cluster analysis, specifying a three-cluster structure, was conducted. This final attempt also failed to validate the cluster structure as it produced 3 clusters with sample sizes very different from the original $(n=5, n=13$, and $n=29$, respectively). Therefore, the cluster structure was deemed insufficient for further use in analyses because it did not produce mean group differences on the Other- 
group factor, it did not produce group differences on two variables that were not utilized in the cluster analysis, it did not replicate using other agglomerative techniques, and a Kmeans cluster analysis failed to replicate group composure.

For purposes of further analyses, groups were divided into the four acculturation strategies based on a median splits from the four factors of the MEIM and Other-group Orientation scale. Although the median split method based only on the MEIM and Other-group Orientation scale was not optimal, it did allow for a larger sample of Latino participants $(n=93)$ to be included in further analyses compared to the sample size $(\mathrm{n}=$ 47) resulting from cluster analyses. Refer to Table 11 for subsample sizes within each acculturation strategy.

\section{Hypothesis Testing}

Hypothesis 1.1: Adolescents characterized as assimilated or marginalized, compared to integrated or separated, are more likely to belong to the juvenile sex offender group.

Hypothesis 1.2: Adolescents characterized as integrated or separated, opposed to assimilated or marginalized, are more likely to be affiliated with the juvenile comparison group.

A 2 X 2 chi-square analysis was conducted to analyze hypothesis 1.1 and hypothesis 1.2. For the initial analysis of hypotheses 1.1 and 1.2, the assimilated and marginalized as well as the integrated and separated groups were collapsed. Results of the initial chi-square analysis indicated that there was no significant relationship between 
acculturation strategy and adolescent group affiliation, $\chi^{2}(1, N=93)=2.91, p=.09$. Moreover, the probability of being in the JSO group and using the assimilation or marginalization acculturation strategies was not statistically different from the probability of being in the JSO group and using the integration or separation strategies. However, the effect size of the chi-square analysis was relatively small $(\Phi=-.18)$. The small effect size was most likely the result of a very small sample size of assimilated and marginalized participants $(n=6)$. Furthermore, there were large discrepancies between cell sizes due to the large difference in acculturation strategy sample sizes ( $n=6$ for assimilated/marginalized versus $n=87$ for integrated/separated).

Although the analysis was non-significant, it is interesting to note that all participants categorized as assimilated or marginalized were also part of the JC group, which was contrary to the research hypotheses. The integrated and separated category was also split, although not evenly, between the two adolescent groups. Refer to Figure 10 for a bar chart of the group assignments. Since the initial, hypothesized relationships were found to be non-significant, no further analyses evaluating differences between the assimilated and marginalized as well as the integrated and separated acculturation strategies were conducted.

Hypothesis 2: Acculturation will mediate the predictive nature of supervisor relationship quality on the juvenile group membership.

In order to determine if acculturation mediated the relationship between supervisor relationship quality and juvenile group affiliation, a series of logistic regressions, using Baron and Kenny’s (1986) causal steps approach to mediation, was 
utilized. Originally, it was proposed that a single measure of supervisor relationship quality would serve as the IV; however, CFAs on the Latino subsample did not support the use of one composite score. Therefore, four of the five factors indicative of supervisor relationship quality (i.e., those identified through the EFA and CFA; General Communication, Daily Communication, Personal Communication, and Attitudes) were used as the independent variables (IV). The fifth factor relating to supervisor quality (Activities) was excluded from analyses because the fit indices from the CFA suggested poor model fit. Before regression analyses were conducted, boxplots on each of the four IVs were assessed in order to identify potential outliers. No outliers were identified in the Daily Communication and Personal Communication variables. There was one outlier in the General Communication variable. This outlier belonged to the JC group and reported a score of 0 , indicating no communication on general life issues. This participant did indicate communication with his supervisor on other variables, so this participant was included in analysis as an important source of variability. Two participants reported relatively low scores (non-zero) on the Attitudes variable and were identified as outliers. These participants represented the two adolescent groups (JSO and JC) and were not identified as outliers in any other supervisor relationship quality variable; therefore, they were included in analyses as important sources of variability. A total of 102 participants were included in the mediated regression analysis.

In the first step of the mediated regression analysis, four separate logistic regressions were conducted. Each of the four supervisor relationship quality indicators (General Communication, Daily Communication, Personal Communication, and 
Attitudes) served as the IV for each logistic regression analysis. The dependent variable (DV) in the first step of the mediated regression analysis was juvenile group membership (JSO and JC). Refer to Table 12 for logistic regression results for each indicator (i.e., factor) of supervisor relationship quality. The relationships for General Communication, Daily Communication, and Attitudes were negatively related to the $\log$ (odds) of being in the JSO group. The personal communication variable was positively related to the $\log$ (odds) of being in the JSO group. However, none of these relationships were statistically reliable. Furthermore, results indicated that none of the four factors of supervisor relationship quality were significantly related to the $\log ($ odds) of being in the JSO group. The relationship between General Communication and the log(odds) of being in the JSO group was not significant, Wald Z $(1)=1.13, p=.29$. Daily Communication was also not significantly related to the $\log$ (odds) of being in the JSO group, Wald $\mathrm{Z}(1)=.37, p=.55$. The relationship between Personal Communication and the $\log$ (odds) of being in the JSO group was non-significant, Wald Z(1) $=.60, p=.44$. Finally, Attitudes was not significantly related to the $\log$ (odds) of being in the JSO group, Wald $\mathrm{Z}(1)=1.28, p=.26$. Moreover, the probability that Latino JSOs reported a low indicator of supervisor relationship quality was the same as that for Latino JCs. Similarly, Latino JCs were no more likely to report a high indicator of supervisor relationship quality than Latino JSOs.

Since the first step in the mediated regression analysis was non-significant on all four of the supervisor relationship quality indicators, further analyses were not warranted. In this case, the meditational nature of acculturation on the relationship between 
supervisor relationship quality and juvenile group membership for Latinos was inconclusive. 


\section{Discussion}

The purpose of this study was to better understand the relationship between family relationships (i.e., supervisor relationship quality), acculturation strategies, and the perpetration of CSA in a population of Latino adolescents. Specifically, this study investigated the types of acculturation strategies used by juvenile sex offenders (JSO) and juvenile comparisons (JC). The data did not support the anticipated hypothesis that Latino JSOs were more likely to endorse an assimilation or marginalization acculturation strategy compared to JC. The hypothesis that Latino JCs, compared to Latino JSOs, were more likely to employ an integrated or separated acculturation strategy was also not supported.

This study also sought to examine the meditational role of acculturation on the relationship between supervisor relationship quality and juvenile group membership (JSO versus JC). The first step of the mediated regression analysis, evaluating the relationship between supervisor relationship quality and juvenile group membership, was nonsignificant for all indicators of supervisor relationship quality. Therefore, further analyses were not warranted, and the meditational role of acculturation could not be determined.

\section{Acculturation Strategy and Juvenile Group Affiliation}

The hypotheses predicting that an assimilated or marginalized Latino adolescent was more likely to belong to the JSO group than the JC and that an integrated or separated Latino adolescent was more likely to belong to the JC than the JSO was not 
supported. Although this hypothesis has not been investigated in a population of juvenile sex offenders, results are contrary to existing literature on juvenile delinquents. Research suggests that Latino adolescent delinquency is associated with assimilation whereas a decreased likelihood of delinquency is related to separation and integration (Berry, 2002; Buriel, Calzada, \& Vasquez, 1982; Fridrich \& Flannery, 1995; Vega, Gil, Warheit, Zimmerman, \& Apospori, 1993). Literature on acculturation suggests that Latinos who assimilate often lose some aspects of their ethnic values, for example familism, that can serve as protective factors against engagement in risky behavior (Wall, Power, \& Arbona, 1993). Similarly marginalized Latino youth oftentimes find themselves without the necessary support for healthy development (Portes \& Rumbaut, 2001). Latinos who are able to maintain their cultural values (i.e., separation) or are able to adapt to the environment based on the cultural setting (i.e., integration) are situated in a better position to utilize available resources, including the family and the community, which may protect against negative and/or unhealthy development (Berry, 2002; Portes \& Rumbaut, 2001).

Multiple factors concerning the data may help to explain why results did not support the study hypotheses. First, results from the cluster analysis, the optimal method to categorize participants into the four acculturation strategies (Phinney, personal communication, October 30, 2009), were not interpretable in this sample. It is possible that the measures utilized were not appropriate or questionnaire presentation was unclear for many of the Latino participants. The Spanish Language Usage factor structure was not supported by CFA results, and the Other-group factor was highly correlated with two 
of the three ethnic identity factors (Belonging and Ethnic Group Clarity). Poor reliabilities for two of the ethnic identity factors (Ethnic Group Clarity and Active) were also indicative of problems with the questionnaire. These problems may involve participant reactivity to the time it takes to complete the various questionnaires. Moreover, the Demographics Questionnaire, which contains the Language Usage Scales as well as the MEIM and Other-group Orientation scale were typically presented last in the sequence of three questionnaires. It is possible that, by the time participants reached the final packet, they were tired and did not provide thoughtful responses. This may have particular relevance for those participants who either were not familiar with Likert-type questionnaires or struggled with other aspects of questionnaire comprehension.

Second, instead of cluster analysis, a series of median splits on the four factors of the MEIM and Other-group Orientation scale were conducted to categorize participants into the four acculturation strategies. Since the Spanish Language Usage factor structure was not supported through the CFA, neither of the Language Usage composite scores were used in the categorization process. However, the Language Usage scores provide important information, and without them, the classification of acculturation strategies is limited (Phinney, personal communication, October 30, 2009).

Another potential explanation for null research findings is that acculturation is based on many more factors than were measured and available for data analysis. As previously mention, acculturation is multidimensional process (Berry, 2001). Latino youth navigating the process are influenced by factors that relate to their parents' context of reception into the U.S., to the societal norms and values, to governmental immigration 
policies, to the coethnic community, as well as to the family (Portes \& Zhou, 1993). These factors influence an acculturation process that occurs differentially among various groups of second-generation immigrant youth. This complex process is what Portes and Rumbaut (2001) call segmented assimilation. For second-generation Latino youth, outcomes of the acculturation process depend on these factors, which are invariably experienced differently for each youth.

For immigrating Latinos (i.e., first-generation), narrow and restrictive immigration policies as well as oppressive and discriminatory values of much of U.S. society create an oftentimes difficult or negative context of reception. Oftentimes, Latinos are forced to reside in marginalized areas, in either inner-city communities or in rural settings. Resources available to settling immigrants, which include social and financial support as well as job opportunities, heavily depend on the size, structure, and location of the community as well as its values and norms (Portes \& Rumbaut, 2001). Community provisions impact family life, especially parents' involvement in their child's life (Portes \& Rumbaut, 2001). The accumulative affects of these factors determine the segmented acculturation pathway of each second-generation Latino. When the community and family are able to support and provide healthy, positive opportunities, Latino youth are better able to follow a positive acculturation trajectory toward upward assimilation (Portes \& Rumbaut, 2001). Upward assimilation, according to Portes and Rumbaut (2001), includes the provision of sufficient economic resources for upward mobility, acquisition of the English language and maintenance of the Spanish language, maintenance of coethnic community values and norms, and development of an 
understanding of American culture. However, when these resources are lacking and when there is strong counterculture negating traditional values and supporting risky behavior, including gang membership and school dropout, Latino youth often spiral down a negative acculturation trajectory toward downward assimilation (Portes \& Rumbaut, 2001). Downward assimilation is characterized by second-generation youth that are isolated from the mainstream culture in their community and/or coethnic cultures, have little to no acquisition of a second language, experience parent-child role reversal, and often suffer from poverty (Portes \& Rumbaut, 2001).

Since acculturation is such a complex and constantly changing process, it is a topic that is difficult to study, even for projects that that set out to specifically investigate the acculturation process. Although attempts were made to collect various acculturation variables, including language usage, ethnic group identification, and identification with other ethnic groups, many factors involved in the acculturation process were not measured. Specifically, there were no measures related to the community in which the participant resided, and no information on cultural and countercultural values was assessed. Furthermore, the categorization of participants into the four acculturation strategies was limited in that it was based primarily on the participants' perceived relationship with his familial supervisor.

Finally, on a conceptual level, juvenile sex offenders may navigate the acculturation process differently than juvenile comparisons. Participants answered questions pertaining to acculturation for the time of measurement, not for the year prior to their incarceration. Some juvenile sex offenders had been part of the juvenile 
correction system for many years. Compared to adolescents living at home, the culture in which juvenile sex offenders are immersed is much different. State-run facilities are extremely structured and rigid, oftentimes inhibiting personal autonomy. Acculturation patterns of juvenile sex offenders may not resemble the acculturation patterns of juvenile comparisons, and the acculturation model utilized in this study may not represent or fully capture the acculturation experiences of incarcerated youth, especially those who have spent years in one or more facilities. Furthermore, non-significant results question the use of Berry's (2001) multi-dimensional model of acculturation. Unfortunately, there has been no research on acculturation studying juvenile sex offenders, and research on acculturation patterns in juvenile delinquents has only utilized unidimensional measures of acculturation (i.e., assimilation to separation or high acculturation to low acculturation). Despite non-significant findings, this study, alone, does not negate future research on acculturation patterns in adolescents with a criminal record, especially since there were a number of methodological limitations impacting the results. Instead, researchers should continue to examine multidimensional models of acculturation with more attention to or broader conceptualizations of acculturation patterns.

Acculturation as a Mediator in the Relationship between Supervisor Relationship Quality and Juvenile Group Affiliation

The second research hypothesis, that acculturation mediated the relationship between supervisor relationship quality and juvenile group membership, was not supported by the data. Moreover, the potential meditational role of acculturation on this 
relationship was not assessed because the relationship between supervisor relationship quality and juvenile group membership was found to be non-significant. These results do not reflect research findings across the field. In fact, studies have unequivocally suggested that poor family relationships serve as a significant risk factor to sexual offending across ethnic groups (Barbaree \& Langton, 2006; Starzyk \& Marshall, 2003; Veneziano \& Veneziano, 2002). Interestingly, the present study found significant differences in mean supervisor relationship quality scores between JSOs and JCs across ethnic groups. When focusing on the Latino subsample, however, these results were not replicated.

The inconclusive results in the first step of the mediated regression analysis, examining the relationship between indicators of supervisor relationship quality and juvenile group affiliation, may have been caused, in part, by the significant differences in reports of the primary supervisor. Descriptive analyses indicated that, for both the JC and JSO groups, the mother was reported as the primary supervisor. However, a chisquare test for independence suggested that Latino JCs were more likely to report being supervised by their mother compared to Latino JSOs. It may be that JSOs, although supervised primarily by the same family member as the JCs, are not supervised as frequently as JCs. This potential difference may have been reflected in this data. In fact, another research project using this same dataset revealed that, across all ethnic groups, JCs report significantly higher supervision quality by their primary caregiver (i.e., their mother) than JSOs (Patterson et al., 2009). Furthermore, research investigating the difference in the frequency of supervision may further inform these inconclusive results. 
As previously mentioned, it is possible that the measures were not well suited for the Latino sample population. Although a CFA supported the Supervisor Relationship Quality factor model for the entire sample population, it was not supported for use in analyses with the Latino subsample. Instead, four of the five first-order factors relating to supervisor relationship quality were used in analyses. The fifth first-order factor was also not supported by a CFA and was not used in the mediated regression analysis. However, only one factor, Daily Communication, was found to have inadequate or poor internal reliability, suggesting that items did, in fact, consistently measure the same construct.

Poor model fit may also be explained by the subsample size of the Latino sample population. The sample size was small for factor analyses $(n=104$; complete data $n=$ 68), decreasing the power of analyses and making significant results difficult to achieve. However, the sample size was sufficiently large to detect significant relationships in the logistic regression analysis, the first step in the mediated regression analysis. Despite this, the effect sizes for each of the four logistic regression analyses were extremely small, explaining only .1 to .2 percent of the variance in the outcome (log(odds) of being in the JSO group).

It is also possible that the type of juvenile offender facility impacted the results of this study. Although all juvenile sexual offenders were known to have committed a sexually related crime, the treatment facilities in which they were collected varied. Moreover, some juvenile offenders were residence of high security juvenile offender facilities run by each state. Other juvenile offenders were living at home or in a community-based home and attended out-patient treatment programs for juvenile sexual 
offenders. Acculturation patterns may differ between participants in these treatment settings. Juvenile offenders located in a state-run facility may be more likely to encounter youth with diverse ethnic backgrounds compared to offenders living at home or even in a specific community-based home. Furthermore, the culture in which they are immersed may look different in a state-run juvenile offender facility than their coethnic community at home. 


\section{Limitations}

It should first be noted that this study is limited in its implications because it utilized archival data to analyze the research questions. Moreover, research questions were derived from known variables within the dataset. Therefore, it can be argued that the available data dictated the applicability and measurement of various theories (e.g., acculturation). Despite this limitation, questionnaires were developed to include common and reliable measures (i.e., MEIM and Other-group Orientation scale) within the field, decreasing potential threats to the validity of the study design.

There are other inherent methodological limitations to this study that may have impacted the measurement and generalizability of the findings. One limitation concerns the internal validity of this study. The non-experimental nature of the proposed study threatens internal validity. More specifically, the lack of randomization of the sample population and random assignment to groups, renders causal inferences inappropriate. Juvenile sex offenders were chosen because they had already been identified within the criminal justice system. It is virtually impossible to access random samples of all offender populations because many cases of CSA remain unreported (Jones \& Finkelhor, 2001). It is also impossible to randomize group treatment because offenders are characterized by their criminal background. Therefore, the non-experimental design of the proposed study is best fit for the research questions.

Additionally, this study focuses on only a sample of identified sex offenders. This group may not be representative of offenders who have not been identified. Implications cannot be made across all juvenile offender populations. As previously 
mentioned, CSA is under reported (Jones \& Finkelhor, 2001), making it difficult to generalize findings to offenders that have not been detected by the criminal justice system. Despite the limited generalizability across all juvenile offender populations, this study utilized a large sample of sex offenders, collected across five different states. The large sample increases the applicability of the findings to the specific population of juvenile sex offenders that are involved in the criminal justice system.

Despite broad participant recruitment, the sample size of Latinos was too small for certain analyses conducted in this study. For instance, when factor model structures are poorly identified (e.g., estimates of means and intercepts were used in this study due to incomplete data) sample sizes for factor analyses even between 400 and 800 may not be sufficient (Wegener \& Fabrigar, 2000). Since the entire sample consisted of 523 participants and only 104 self-identified Latinos, factor analyses were limited. Statisticians have also suggested that factor analyses are cross-validated for maximum validity assurance (McDonald, 1999); however, the samples were not large enough to split between the two factor analyses, let alone cross-validate each one. More problematic was that the majority of Latino participants did not have complete data, which may have been indicative of participant reactivity (e.g., disinterest, boredom, confusion, or fatigue). Incomplete data affected multiple analyses, including all factor analyses and both methods of determining acculturation strategies (i.e., cluster analysis and median splits), which ultimately limited the sample sizes for analyses concerning the hypotheses. A larger Latino sample size could increase the probability of finding significant results. At the very least, a larger Latino sample size would allow for cross- 
validation of factor analyses increasing the likelihood of finding a good factor model fit to the data. Categorization of participants into the four acculturation strategies may also be more interpretable with a larger sample size and a more complete dataset.

Laws surrounding sexual abuse perpetrated by immigrants may have created some selection bias or played a role in the opportunities, or lack thereof, to collect data from Latino participants. Federal laws require deportation of a Latino adolescent, if arrested for sexual offense. Although individual states are responsible for creating and implementing laws regarding sexual offending, the Illegal Immigration Reform and Immigration Responsibility Act of 1996, which is a set of federal laws, allows state officials to deport undocumented as well as documented immigrants for crimes involving "moral turpitude" that would justify a one-year sentence. Sexual offending against a child is included as a crime warranting deportation in this Act, no matter the perpetrator's age. Deportation may occur despite one's legal status; therefore, a Latino adolescent with a VISA or other legal documentation who is convicted of a sex crime is subject to deportation. Since deportation is the legal outcome of sexual offending for many Latino adolescent perpetrators, it may be difficult to obtain a substantial, representative sample of Latino juvenile sexual offenders.

Latino cultural norms may impact the generalizability of the sample population. For many Latinos, sexual abuse is a taboo topic (Fontes et al., 2001). Along with this, cultural values of shame and family connectedness may result in under reporting of CSA by Latino communities (Bacigalupe, 2001). Furthermore, maintenance of these cultural norms may limit the discussion and reporting of sexual offending. Therefore, the 
generalizability of these study findings may be limited among various Latino populations where sexual abuse is a taboo.

Similarly, this study is limited in its generalizability across all Latino populations in the U.S. Latinos are a heterogeneous group, emigrating from many different countries of origin, speaking numerous languages, and acculturating in different ways (Bacigalupe, 2001; Coatsworth et al., 2005). The diversity of locations of data collection may have helped as well as hindered the generalizability. As previously mentioned, data was collected in five different states. States from which data was collected were meant to represent people from a wide geographic range in the U.S. Although the diversity obtained from data collection across the U.S. augments generalizability, the Latino cultural background represented within each state may be significantly different. For instance, Latino participants residing in Florida may be more likely to have immigrated from Puerto Rico or Cuba, whereas those from Texas may more likely be from Mexico. Therefore, a more focused approach investigating a specific sub-population of Latinos (e.g., Mexican-Americans in Oregon) could help to address the heterogeneity among Latino groups. However, preliminary data focusing on understudied populations like Latinos in the U.S. serves to create a foundation for population-specific research and culturally sensitive prevention.

Finally, CSA is a sensitive topic for everyone involved, including the perpetrator. Data was collected using self-report questionnaires, and some juveniles may have felt uncomfortable sharing the details of their offenses. Despite attempts to assure participants that the data were collected in an anonymous fashion, some may have 
hesitated to provide full disclosure or may not have been truthful in their responses. Like many self-report questionnaires, data are limited to the responses given by each

participant. However, self-report questionnaires can provide equally valid or more valid information from juvenile sex offenders compared to interviews, official reports, or other methodological procedures (Kaufman, Hilliker, Lathrop, Daleiden, \& Rudy, 1996;

Krohn, Waldo, \& Chiricos, 1974; Elliot \& Ageton, 1980). Self-report measures have also been found to be useful in gaining reliable information from adolescent sex offenders' patterns of perpetration or modus operandi (Kaufman, Hilliker, Lathrop, \& Daleiden, 1993; Kaufman et al., 1996). 
Implications and Future Directions

The proposed study hypothesized that acculturation strategy would be related to juvenile group affiliation (JSO versus JC). It was also hypothesized that acculturation strategy mediates the relationship between supervisor relationship quality and adolescent group membership. Although the data did not support these hypotheses, there were several problematic issues that may have lead to inconclusive results. Nevertheless studies like this one could have significant implications for sex offenders' assessment and treatment as well as community-based prevention efforts.

Only within the last two decades have treatment programs for juvenile sex offenders moved beyond a “one-size fits all” approach (Langton \& Barbaree, 2006). Findings that indicate that some sex offender populations maintain different or diverse cultural values may support the notion that treatment programs should take a more individualized and culturally sensitive approach. In fact, significant results could have suggested that treatment programs should transition to a population-specific approach taking into account all culturally relevant factors. It may be evident that, even within narrowed populations, it is difficult to profile offenders for treatment purposes due to varying acculturative strategies. In this case, interventions may need to evaluate offenders on a case-by-case basis, making treatment more tailored to an offender's history, acculturation strategy, and relationship with family members and/or supervisors.

Differences in acculturation strategies may impact the etiology of offending behavior as well as adolescents' perception and success within offender treatment programs. Knowledge of specific cultural orientation may help guide practitioners to 
develop appropriate case plans. For example, a Latino adolescent who is oriented more toward his cultural heritage may interpret components of offender treatment programs (e.g., sexual education, sexual scripts) differently than a Latino adolescent who is oriented more toward American culture. This same Latino adolescent may also face different risks factors (e.g., gangs, school dropout) when he returns to his family and community, particularly if he is assimilated or marginalized. These factors should be incorporated into the community transition process and safety planning procedures as part of the treatment process.

Knowing whether an offender and his family maintain high quality or close-knit relationships may also help practitioners intervene on more than an individual level. Incorporating family support into treatment, particularly for those that would benefit from it, may help decrease recidivism for both sexual and non-sexual crimes. Treatment that involves family members may also have restorative elements that reunite families or increase the cohesion between family members, especially if there were tensions prior to or following the adolescent's incarceration (e.g., in intra-familial cases of sexual abuse).

Another important implication for this field of research is its potential to inform prevention efforts. Findings that indicate that Latino juvenile sexual offenders experience or utilize a specific acculturation strategy could help inform prevention programs that target Latino communities. Although the acculturation process is not unique to Latinos, there are common factors that influence this process among Latino adolescents. Identification of these factors may guide the development and implementation of prevention programs. For example, results that suggest that 
(downward) assimilation serves as a risk factor for sexual offending may help psychologists, other practioners, and community members focus attention on Latino adolescents who seem to be on this downward trajectory. What may be more beneficial than taking an individual level approach to prevention, however, is to focus community attention on promoting positive, healthy engagement in the coethnic community and the family. The value of research similar to this study is to uncover the differential risk factors of sexual offending so that they can be incorporated into prevention efforts within specific communities.

Future research should continue to take a population-specific approach to studying problematic social issues like CSA. There are several design suggestions that may help improve the nature of the study results. First, as previously mentioned, researchers should focus more attention on the conceptualization and measurement of acculturation in juvenile sex offenders, especially since acculturation experiences of juvenile offenders may differ from adolescents without a criminal history. Second, investigators should recruit a sample size large enough to satisfy the minimal requirements of the particular statistical analyses intended to be used. Replicating the analyses attempted in this study would involve the inclusion of a sample adequate to meet the demands of the factor analytic and cluster analysis techniques. Furthermore, power analyses should be conducted to identify the same size needed for each analysis. Third, researchers should couple quantitative datasets with qualitative investigations on a more specific subpopulation of Latinos, for example Mexican Americans in Oregon. Doing so could help to narrow an extremely heterogeneous population like Latinos. A qualitative 
or case study design could help researchers better describe and understand the burgeoning field of research on acculturation, cultural issues, and CSA. It will also be important for future research on acculturation and CSA to consider all variables, at multiple levels of analysis that impact the acculturation process. Key factors to study would include cultural value and norms associated with the community in which the participants reside as well as societal values and norms, governmental policies, and other contexts of reception for second-generation Latino youth.

Finally, it is important to mention that population-specific and cross-cultural research can be a difficult to interpret, especially for publication. In this line of research, there is danger in interpreting results that can be damaging to different cultures. However, results should never implicate negative aspects of any culture. Furthermore, rather than identifying risk factors uniquely related to a specific ethnic group, research that pursues the examination of culturally relevant variables of sexual offending should identify strengths within and between cultures that may help inform intervention and prevention efforts in various communities. 
Figure 1.

Multidimensional Model of Acculturation

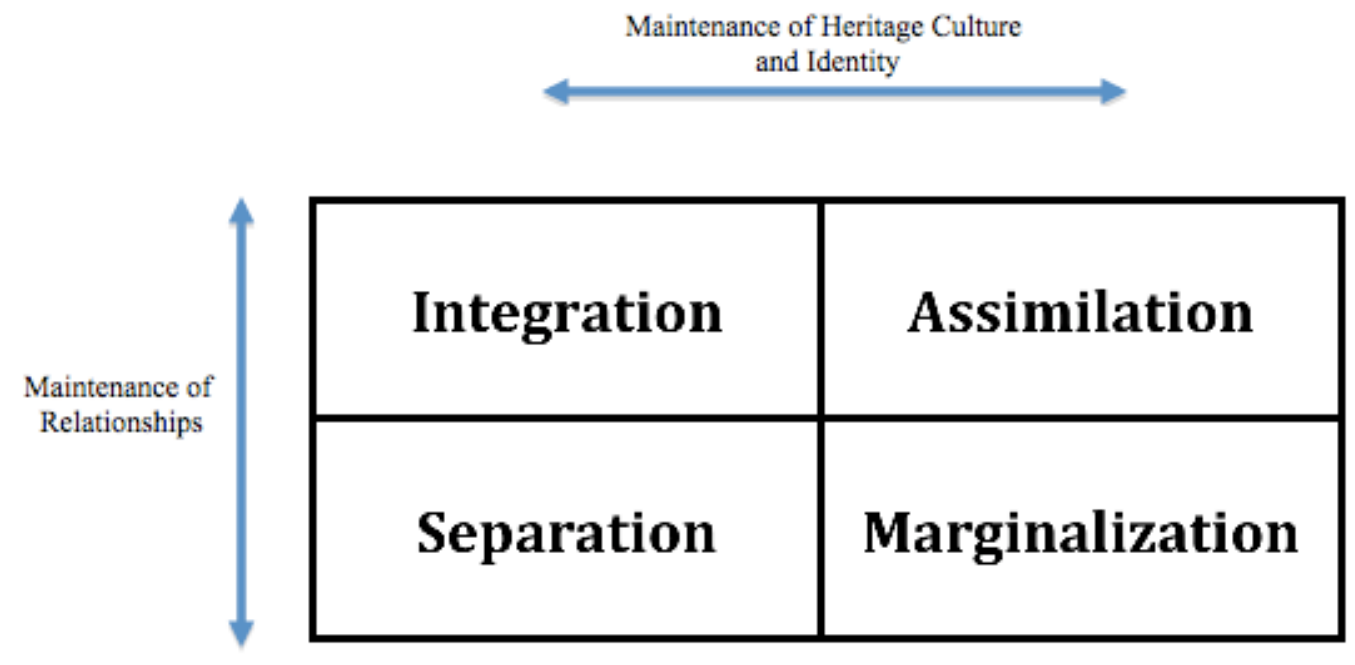

*Adapted from Berry (2002; 2001) 
Figure 2.

Mediated Model for Hypothesis 2

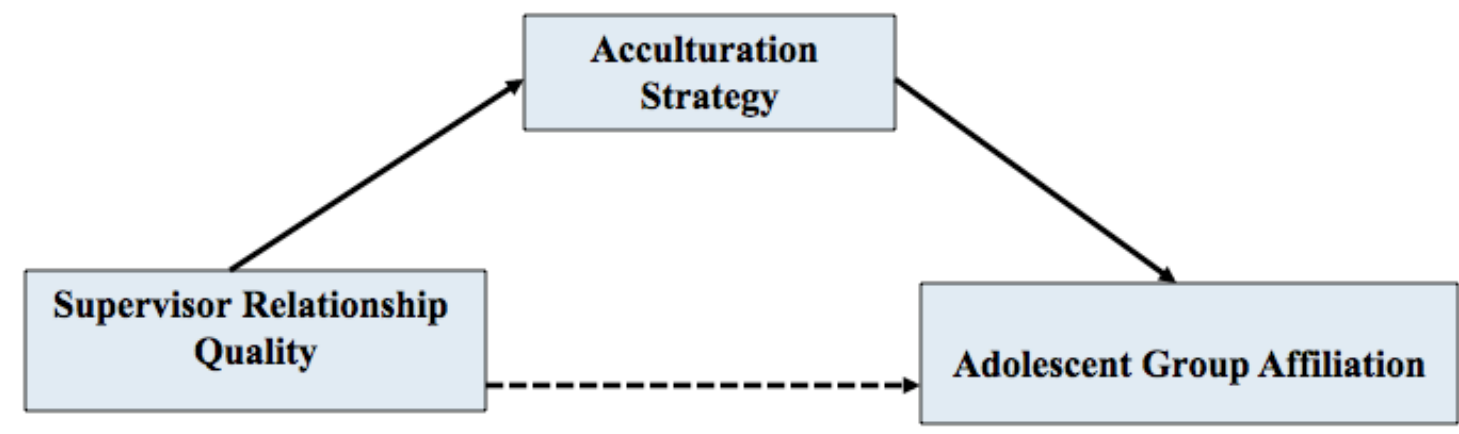


Figure 3.

Line Graph of Interaction between Grade Level Completion and Ethnicity

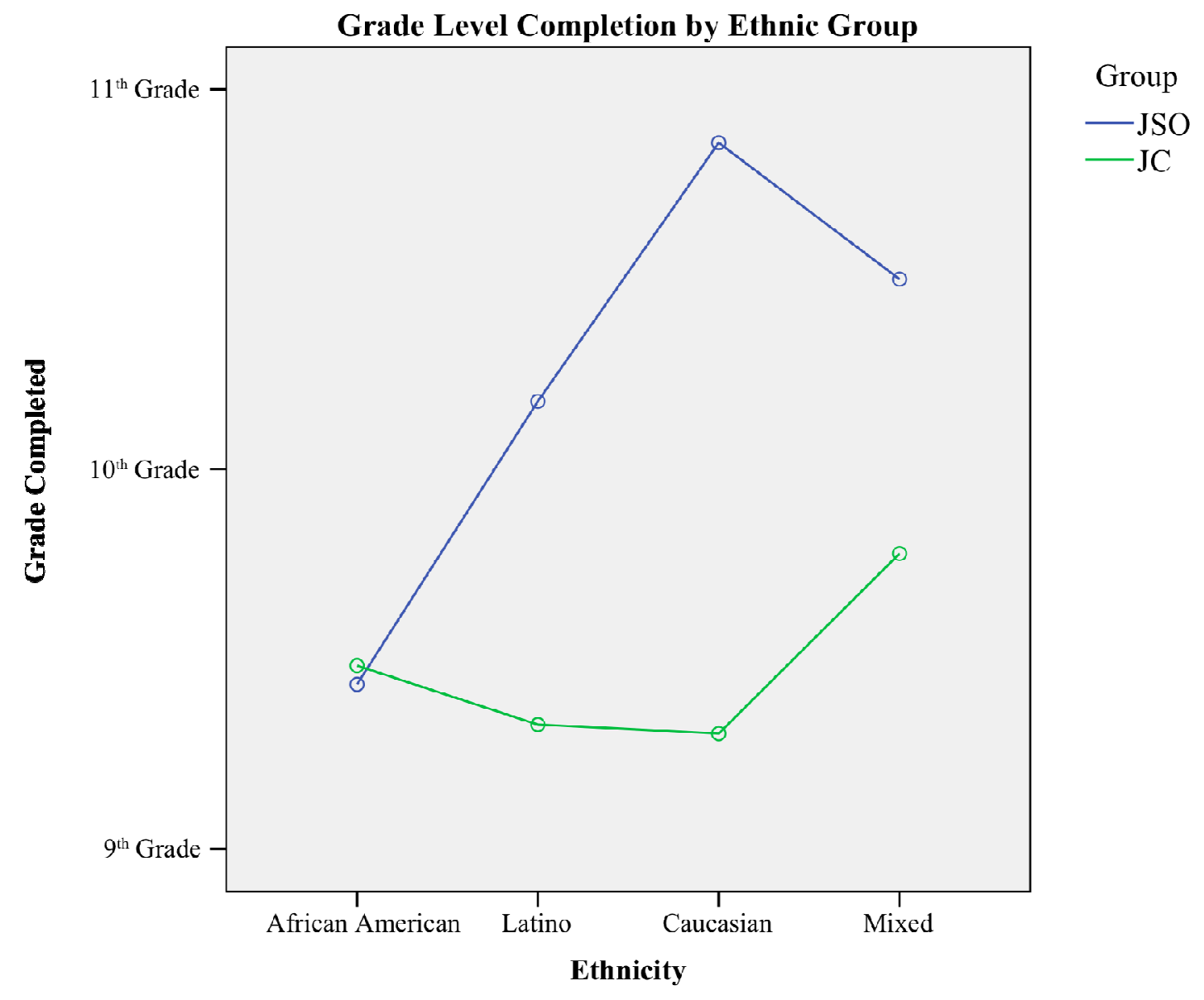


Figure 4.

Factor Model for Supervisor Relationship Quality

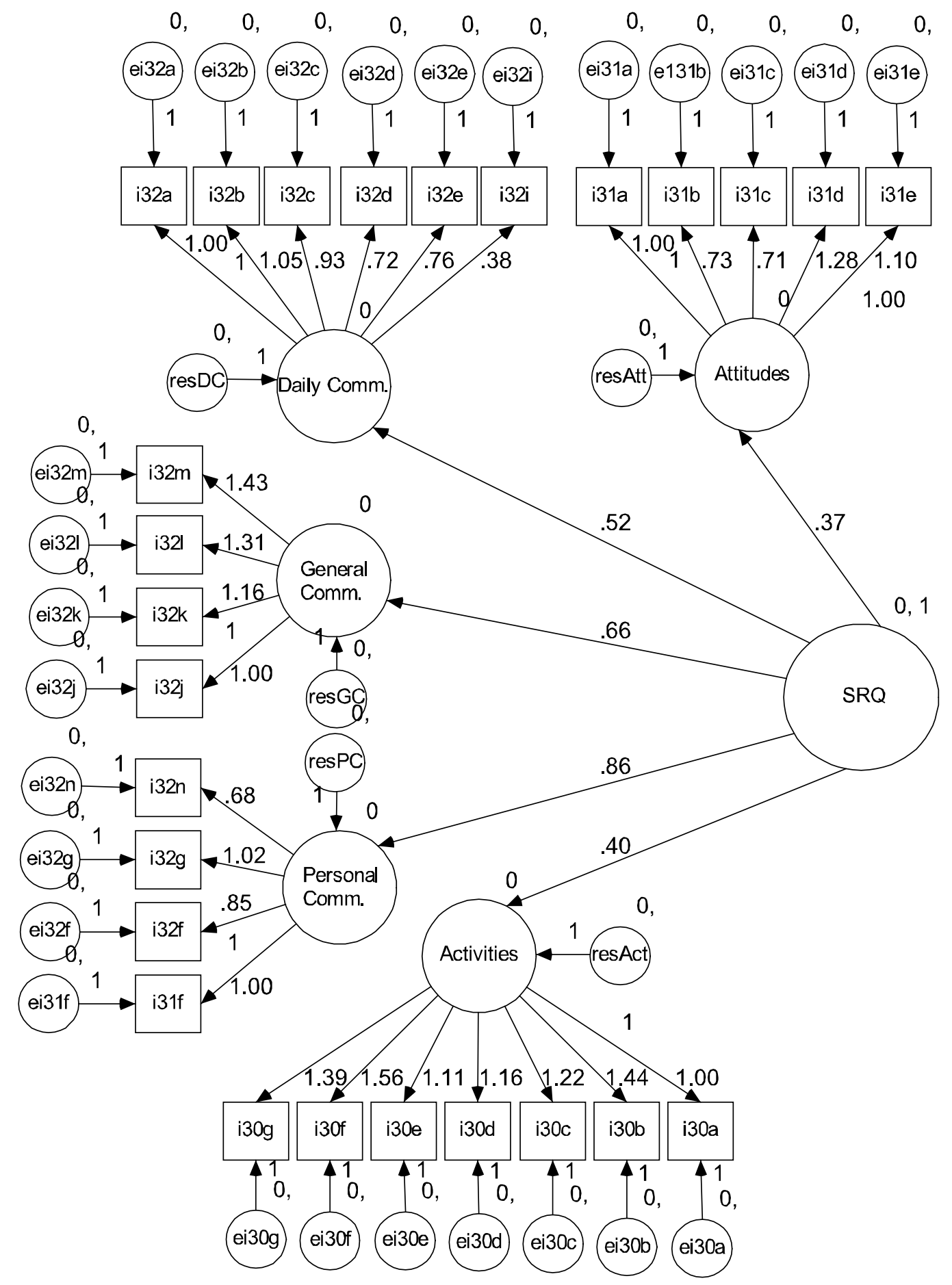

* All items can be found in Appendices B, C, and D. 
Figure 5.

Factor Model for Supervisor Relationship Quality - Improvement for Latinos

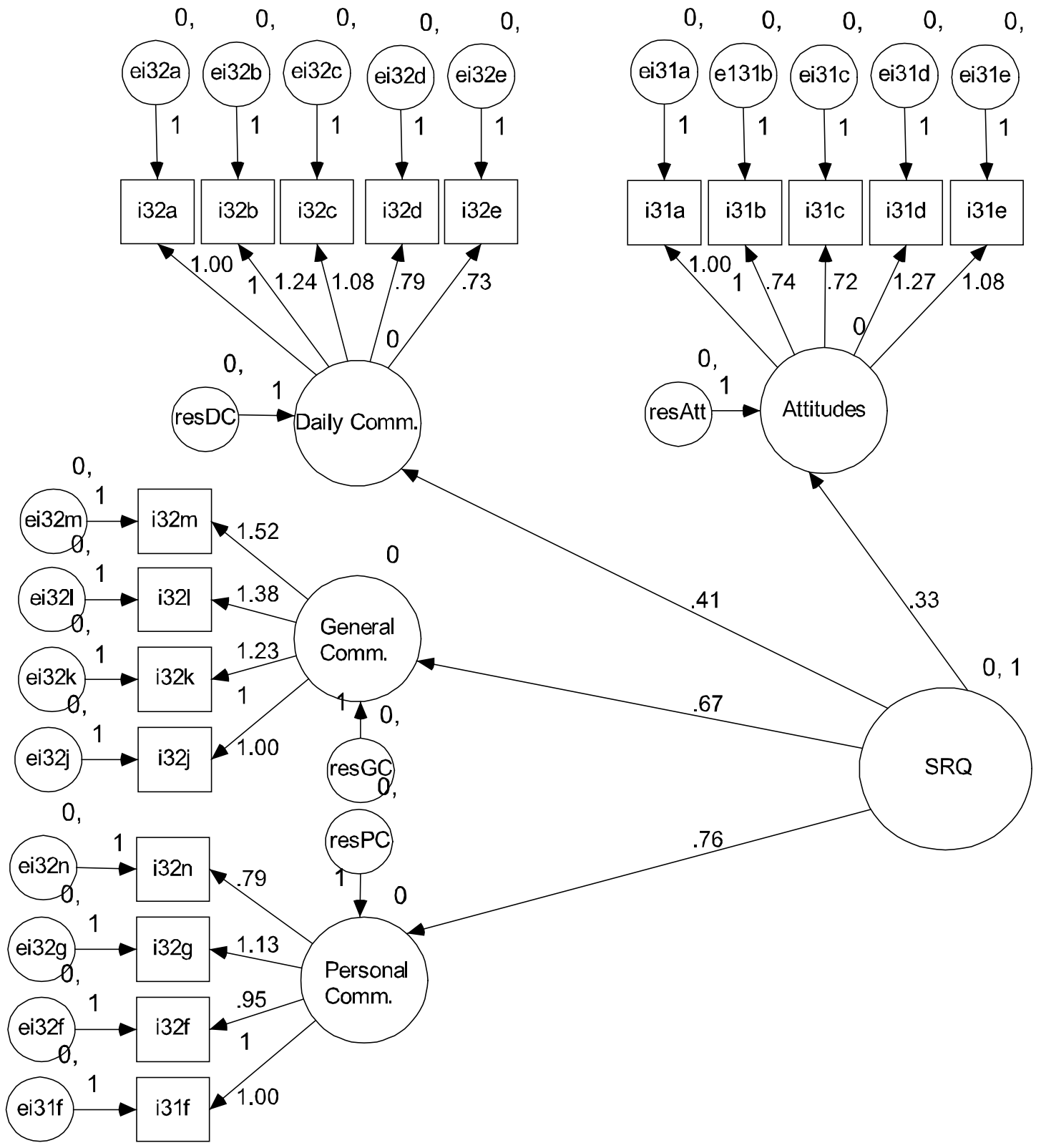

* All items can be found in Appendices B, C, and D. 
Figure 6.

\section{Factor Model for English Language Usage}

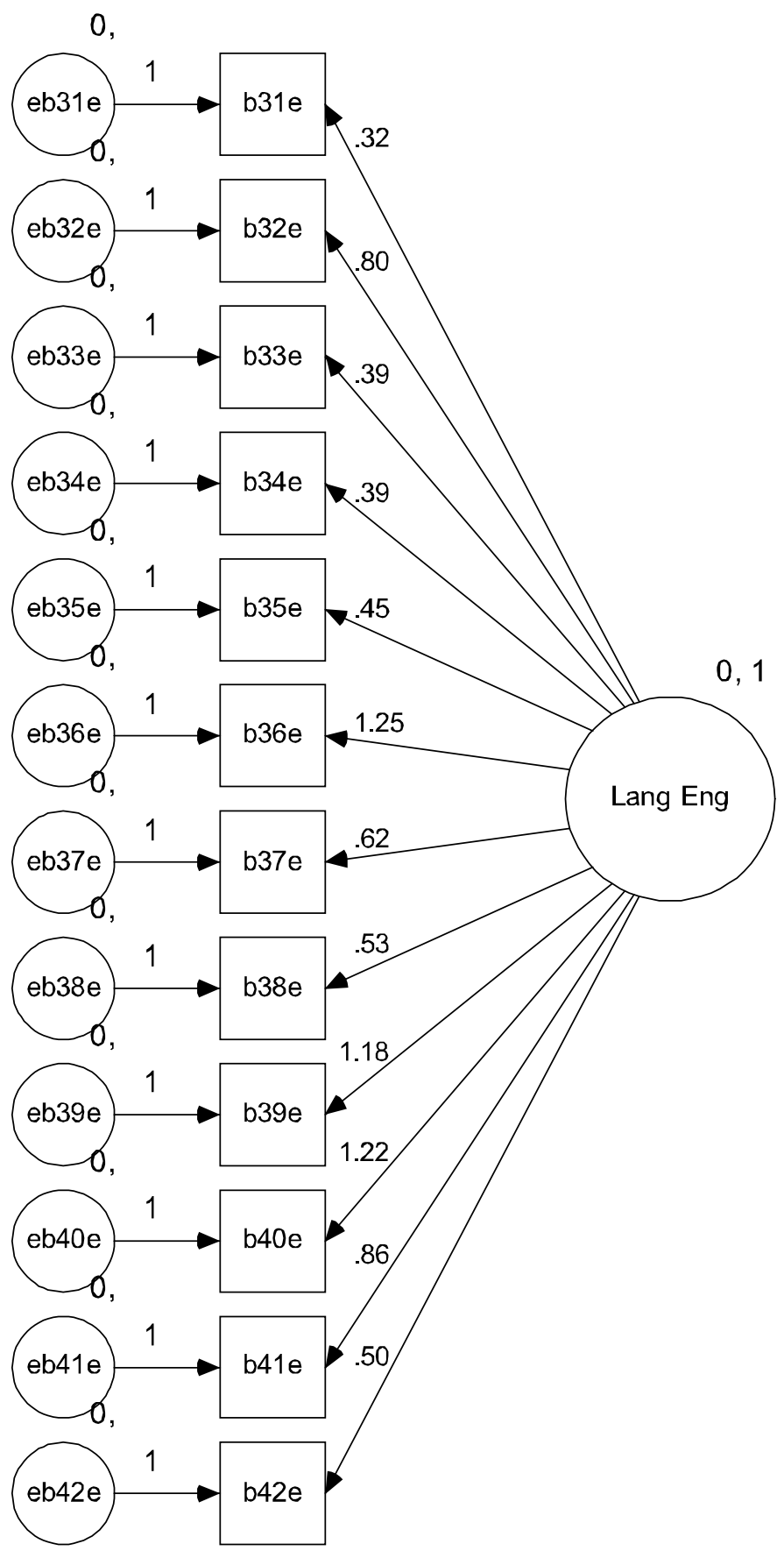

* All items can be found in Appendix F. 
Figure 7.

Factor Model for Spanish Language Usage

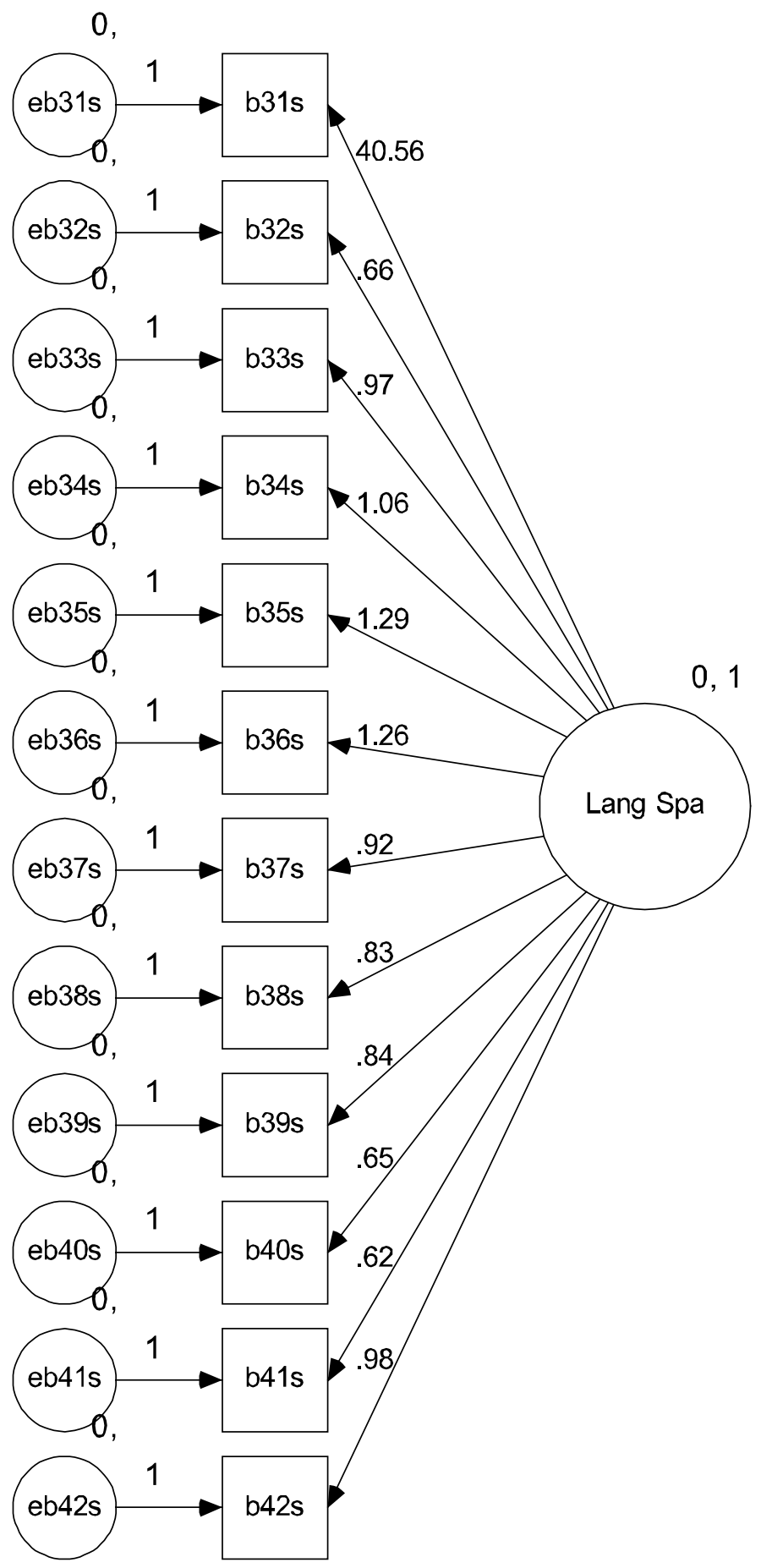

* All items can be found in Appendix F. 
Figure 8.

Factor Model for MEIM and Other-group Orientation

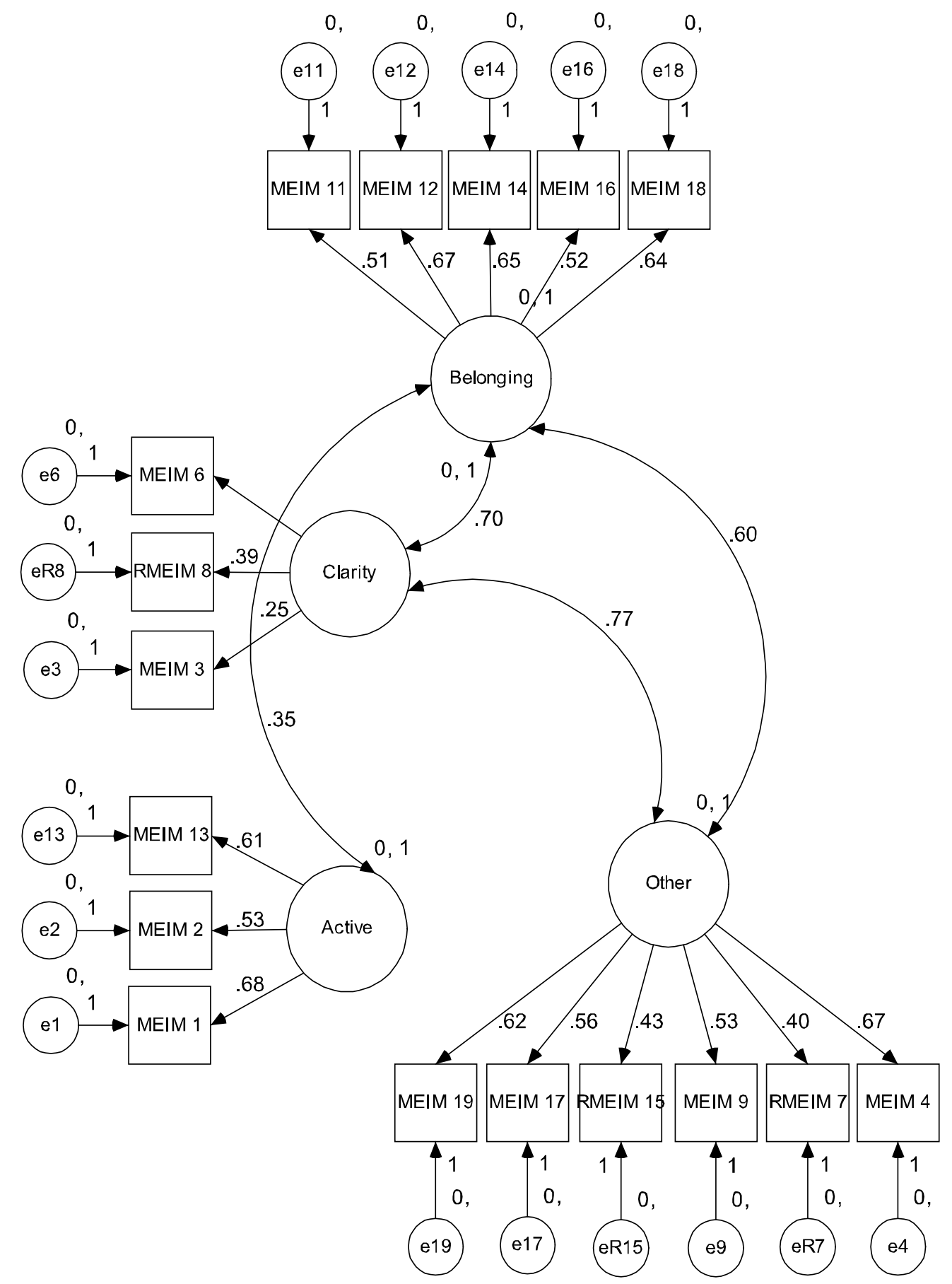

* All items can be found in Appendix E. 
Figure 9 .

Dendogram for Acculturation Strategy

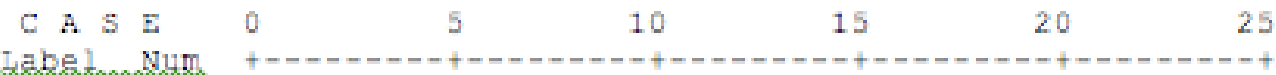

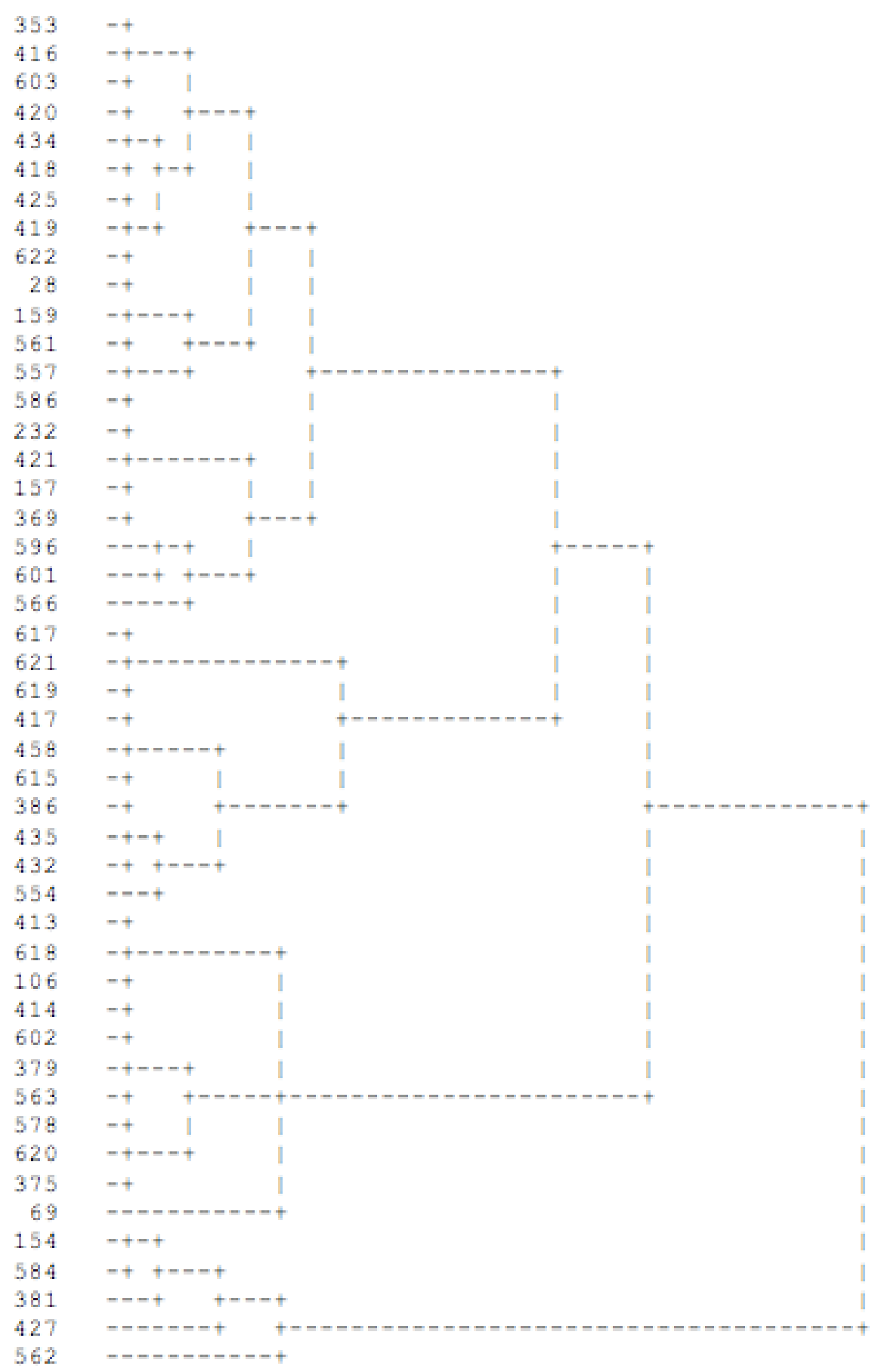


Figure 10.

Bar Chart of Group Assignments for Hypothesis 1

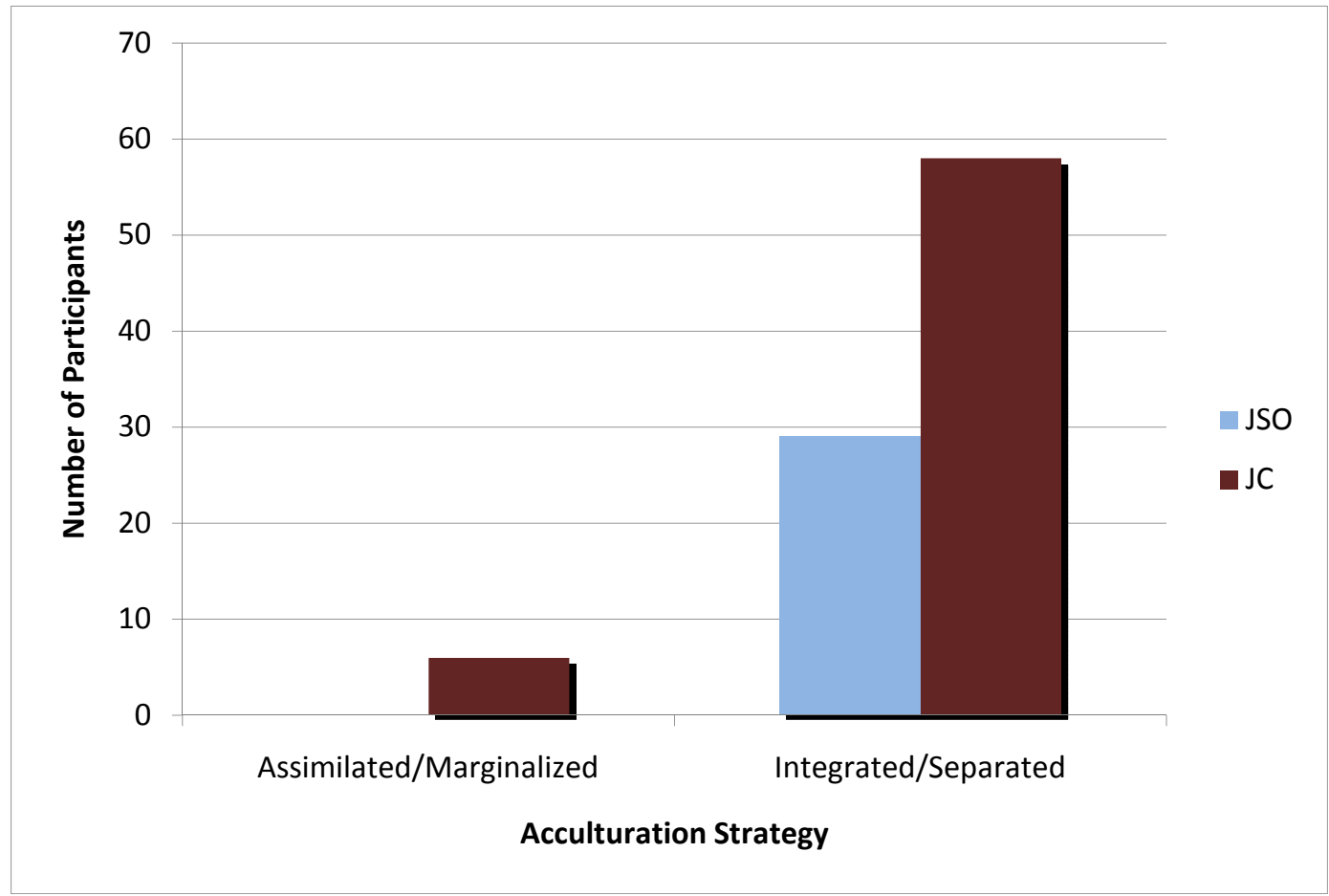


Table 1.

Participants' Group Affiliation and Self-Reported Ethnicity

\begin{tabular}{|c|c|c|}
\hline $\mathrm{N}=523$ & $\mathrm{JSO}(\%)$ & $\mathrm{JC}(\%)$ \\
\hline African American & $34(6.5)$ & $68(8.4)$ \\
\hline Caucasian & $159(30.4)$ & $85(13.0)$ \\
\hline Latino & $33(4.1)$ & $71(13.6)$ \\
\hline Mixed & $52(6.3)$ & $21(4.0)$ \\
\hline
\end{tabular}


Table 2.

Frequencies of Participants from Each Ethnic Group by State

\begin{tabular}{|l|c|c|c|c|c|}
\hline Ethnicity & FL & OR & NY & SC & TX \\
\hline $\begin{array}{l}\text { African } \\
\text { American }\end{array}$ & 21 & 19 & 16 & 25 & 21 \\
\hline Caucasian & 18 & 162 & 3 & 50 & 11 \\
\hline Latino & 5 & 26 & 46 & 1 & 26 \\
\hline Mixed & 10 & 49 & 3 & 6 & 5 \\
\hline Total & 54 & 256 & 68 & 82 & 63 \\
\hline
\end{tabular}


Table 3.

Sample Sizes for Analyses

\begin{tabular}{|c|c|c|}
\hline Analysis & Sample Size in Analysis & Original Sample Size \\
\hline $\begin{array}{l}\text { Descript. and Initial } \\
\text { Inferential Anal. for all } \\
\text { Ethnicities }\end{array}$ & $461-523$ & 523 \\
\hline $\begin{array}{l}\text { Descript. and Initial } \\
\text { Inferential Anal. for Latino } \\
\text { subsample }\end{array}$ & 86-104 & 104 \\
\hline $\begin{array}{l}\text { EFA on Super. Relat. } \\
\text { Qual. for all Ethnicities }\end{array}$ & 372 & 523 \\
\hline $\begin{array}{l}\text { EFA on Super. Relat. } \\
\text { Qual. for Latino } \\
\text { subsample }\end{array}$ & 68 & 104 \\
\hline $\begin{array}{l}\text { CFA on Super. Relat. } \\
\text { Qual. for all Ethnicities }\end{array}$ & 523 & 523 \\
\hline $\begin{array}{l}\text { CFA on Super. Relat. } \\
\text { Qual. for Latino } \\
\text { subsample }\end{array}$ & 104 & 104 \\
\hline $\begin{array}{l}\text { EFA on Eng. Language } \\
\text { Usage (Latino subsample) }\end{array}$ & 63 & 104 \\
\hline $\begin{array}{l}\text { CFA on English Language } \\
\text { Usage (Latino subsample) }\end{array}$ & 104 & 104 \\
\hline $\begin{array}{l}\text { EFA on Spanish Language } \\
\text { Usage (Latino subsample) }\end{array}$ & 45 & 104 \\
\hline $\begin{array}{l}\text { CFA on Spanish Language } \\
\text { Usage (Latino subsample) }\end{array}$ & 104 & 104 \\
\hline $\begin{array}{l}\text { Acculturation from Cluster } \\
\text { Analysis (Latino } \\
\text { subsample) }\end{array}$ & 46 & 104 \\
\hline $\begin{array}{l}\text { Acculturation from } \\
\text { Median Splits } \\
\end{array}$ & 93 & 104 \\
\hline Chi-Square (Hypothesis 1) & 93 & 104 \\
\hline $\begin{array}{l}\text { Mediated Regression } \\
\text { (Hypothesis 2) }\end{array}$ & 102 & 104 \\
\hline
\end{tabular}


Table 4.

Mean Age (SD) (Group Affiliation X Self-Reported Ethnicity)

\begin{tabular}{|l|c|c|c|}
\hline Ethnicity & JSO & JC & Totals \\
\hline African-American & $13.71(1.32)$ & $14.75(1.77)$ & $14.40(1.70)$ \\
\hline European-American & $14.19(1.45)$ & $14.55(1.43)$ & $14.32(1.45)$ \\
\hline Latino & $14.12(1.34)$ & $14.41(1.73)$ & $14.32(1.62)$ \\
\hline Mixed Ethnicity & $13.92(1.48)$ & $15.05(1.20)$ & $14.25(1.49)$ \\
\hline Totals & $14.07(1.43)$ & $14.61(1.61)$ & $14.32(1.54)$ \\
\hline
\end{tabular}


Table 5.

Percentage of Top 3 Family Members Providing Supervision (Group X Ethnicity)

\begin{tabular}{|l|c|c|c|c|c|c|}
\hline & \multicolumn{3}{|c|}{ JSO } & \multicolumn{3}{c|}{ JC } \\
\hline & Mother & Father & Grandmother & Mother & Father & Grandmother \\
\hline $\begin{array}{l}\text { African- } \\
\text { American }\end{array}$ & 65 & 35 & 35 & 93 & 44 & 41 \\
\hline & Mother & Father & Grandmother & Mother & Father & Grandmother \\
\hline $\begin{array}{l}\text { European- } \\
\text { American }\end{array}$ & 79 & 49 & 34 & 93 & 74 & 31 \\
\hline & Mother & Father & Aunt & Mother & Father & Aunt \\
\hline Latino & 76 & 58 & 33 & 99 & 69 & 32 \\
\hline $\begin{array}{l}\text { Mixed } \\
\text { Ethnicity }\end{array}$ & Mother & Father & Grandmother & Mother & Father & Grandmother \\
\hline
\end{tabular}


Table 6.

Internal Reliabilities (i.e., Internal Consistencies)

\begin{tabular}{|c|c|c|c|}
\hline Sample & Factor & $\begin{array}{c}\text { Reliability (Cronbach's } \\
\text { Alpha) }\end{array}$ & $\begin{array}{l}\text { Number of } \\
\text { Items }\end{array}$ \\
\hline \multirow[t]{6}{*}{ All Ethnicities } & $\begin{array}{l}\text { General } \\
\text { Communication }\end{array}$ & .80 & 4 \\
\hline & Daily Communication & .77 & 6 \\
\hline & $\begin{array}{l}\text { Personal } \\
\text { Communication }\end{array}$ & .75 & 4 \\
\hline & Activities & .81 & 7 \\
\hline & Attitudes & .84 & 5 \\
\hline & Superv. Relat. Quality & .83 & 5 \\
\hline \multirow[t]{6}{*}{ Latinos } & $\begin{array}{l}\text { General } \\
\text { Communication }\end{array}$ & .82 & 4 \\
\hline & Daily Communication & .65 & 6 \\
\hline & $\begin{array}{l}\text { Personal } \\
\text { Communication }\end{array}$ & .74 & 4 \\
\hline & Activities & .78 & 7 \\
\hline & Attitudes & .81 & 5 \\
\hline & Superv. Relat. Quality & .79 & 5 \\
\hline \multirow[t]{6}{*}{ Latinos } & $\begin{array}{l}\text { English Language } \\
\text { Usage }\end{array}$ & 1.0 & 12 \\
\hline & $\begin{array}{l}\text { Spanish Language } \\
\text { Usage }\end{array}$ & .85 & 12 \\
\hline & Belonging & .78 & 5 \\
\hline & Ethnic Group Clarity & .40 & 3 \\
\hline & Active & .66 & 3 \\
\hline & Other & .75 & 6 \\
\hline
\end{tabular}


Table 7.

Correlation Matrix for MEIM and Other-group Orientation Factors from EFA

\begin{tabular}{|l|c|c|c|c|}
\hline & Belonging & Clarity & Active & Other \\
\hline Belonging & 1 & & & \\
\hline Clarity & .17 & 1 & & \\
\hline Active & .39 & -.06 & 1 & \\
\hline Other & .28 & .27 & -.03 & 1 \\
\hline
\end{tabular}


Table 8.

Correlation Matrix of Six Acculturation Factors

\begin{tabular}{|l|c|c|c|c|c|c|}
\hline & English & Spanish & Belonging & Clarity & Active & Other \\
\hline English & 1 & & & & & \\
\hline Spanish & -.21 & 1 & & & & \\
\hline Belonging & -.12 & -.22 & 1 & & & \\
\hline Clarity & .04 & -.21 & .53 & 1 & & \\
\hline Active & -.09 & -.15 & .25 & .12 & 1 & \\
\hline Other & .20 & -.14 & .50 & .48 & .07 & 1 \\
\hline
\end{tabular}


Table 9.

Means and Standard Deviations of Clusters on Cluster Variables

\begin{tabular}{|l|c|c|c|c|c|c|c|c|c|c|c|c|}
\hline & \multicolumn{2}{|c|}{ English } & \multicolumn{2}{c|}{ Spanish } & \multicolumn{2}{c|}{ Belonging } & \multicolumn{2}{c|}{ Clarity } & \multicolumn{2}{c|}{ Active } & \multicolumn{2}{c|}{ Other } \\
\hline Cluster & $\mathrm{M}$ & $\mathrm{SD}$ & $\mathrm{M}$ & $\mathrm{SD}$ & $\mathrm{M}$ & $\mathrm{SD}$ & $\mathrm{M}$ & $\mathrm{SD}$ & $\mathrm{M}$ & SD & $\mathrm{M}$ & $\mathrm{SD}$ \\
\hline Separated & 2.71 & .10 & 2.32 & .13 & 2.06 & .11 & 1.77 & .11 & 2.42 & .16 & 1.68 & .10 \\
\hline Assimilated & 3.38 & .14 & 1.31 & .17 & 1.55 & .15 & 1.27 & .15 & 2.70 & .22 & 1.50 & .14 \\
\hline Integrated & 3.61 & .15 & 2.76 & .18 & 1.22 & .16 & 1.40 & .16 & 1.83 & .23 & 1.33 & .15 \\
\hline
\end{tabular}


Table 10.

Post hoc ANOVA Results - Significance of Mean Cluster Differences Between Cluster Variables

\begin{tabular}{|l|c|c|c|c|c|c|c|c|c|c|c|c|c|c|c|c|c|c|}
\hline & \multicolumn{3}{|c|}{ English } & \multicolumn{3}{c|}{ Spanish } & \multicolumn{3}{c|}{ Belonging } & \multicolumn{3}{c|}{ Clarity } & \multicolumn{3}{c|}{ Active } & \multicolumn{3}{c|}{ Other } \\
\hline Cluster & $\mathrm{S}$ & $\mathrm{A}$ & $\mathrm{I}$ & $\mathrm{S}$ & $\mathrm{A}$ & $\mathrm{I}$ & $\mathrm{S}$ & $\mathrm{A}$ & $\mathrm{I}$ & $\mathrm{S}$ & $\mathrm{A}$ & $\mathrm{I}$ & $\mathrm{S}$ & $\mathrm{A}$ & $\mathrm{I}$ & $\mathrm{S}$ & $\mathrm{A}$ & $\mathrm{I}$ \\
\hline Separated & - & $.00 *$ & $.00 *$ & - & $.00 *$ & .12 & - & $.03 *$ & $.00 *$ & - & $.03 *$ & .15 & - & .56 & .10 & - & .56 & .14 \\
\hline Assimilated & $.00 *$ & - & .51 & $.00 *$ & - & $.00^{*}$ & $.03 *$ & - & .31 & $.03 *$ & - & .83 & .56 & - & $.02 *$ & .56 & - & .69 \\
\hline Integrated & $.00 *$ & .51 & - & .12 & .00 & - & $.00 *$ & .31 & - & .15 & .83 & - & .10 & $.02 *$ & - & .14 & .69 & - \\
\hline
\end{tabular}

$*$ The mean difference is significant at $p<.05$. 
Table 11.

Subsample Sizes of Acculturation Strategies from Median Splits

\begin{tabular}{|l|c|c|}
\hline Acculturation Strategy & $\mathrm{n}$ & $\%$ \\
\hline Assimilation & 2 & 1.9 \\
\hline Integration & 48 & 46.2 \\
\hline Separation & 39 & 37.5 \\
\hline Marginalization & 4 & 3.8 \\
\hline
\end{tabular}


Table 12.

Results from the First Step of the Mediation Analysis

\begin{tabular}{|l|c|c|c|c|c|}
\hline & $\begin{array}{c}\text { Correlation } \\
\text { Coefficient }\end{array}$ & $\begin{array}{c}\text { Wald Z } \\
\text { Statistic }\end{array}$ & $\begin{array}{c}\text { Prob. (P) of } \\
\text { Wald Z }\end{array}$ & $\chi^{2}$ & Nagelkerke R $^{2}$ \\
\hline $\begin{array}{l}\text { General } \\
\text { Communication }\end{array}$ & -.23 & 1.13 & .29 & 1.14 & .02 \\
\hline $\begin{array}{l}\text { Daily } \\
\text { Communication }\end{array}$ & -.17 & .37 & .55 & .37 & .01 \\
\hline $\begin{array}{l}\text { Personal } \\
\text { Communication }\end{array}$ & .16 & .60 & .44 & .60 & .01 \\
\hline Attitudes & -.33 & 1.28 & .26 & 1.28 & .02 \\
\hline
\end{tabular}




\section{References}

Abel, G.G., Osborn, C.A., \& Twigg, D.A. (1993). Sexual assault through the life span: Adult offenders with juvenile histories. In H.E. Barbaree, W.L. Marshall, \& S.M. Hudson (Eds.), The Juvenile Sex Offender (pp. 104-117). New York: The Guilford Press.

Andrés-Hyman, R.C., Cott, M.A., \& Gold, S.N. (2004). Ethnicity and sexually orientation as PTSD mitigators in child sexual abuse survivors. Journal of Family Violence, 19(5), 319-325.

Arrellano, C.A., Kuhn, J.A., \& Chavez, E.L. (1997). Psychosocial correlates of sexual assault among Mexican American and White non-Hispanic adolescent females. Hispanic Journal of Behavioral Sciences, 19(4), 446-460.

Arroyo, J.A., Simpson, T.L., \& Aragon, A.S. (1997). Childhood sexual abuse among Hispanic and Non-Hispanic White college women. Hispanic Journal of Behavioral Sciences, 19(1), 57-68.

Bacigalupe, G. (2001). Latin@ child sexual abuse survivors in the United States: Relational assessment and intervention. Psyke, 10(2), 167-180.

Baer, J.C. \& Schmitz, M.F. (2007). Ethnic differences in trajectories of family cohesion for Mexican American and non-Hispanic White adolescents. Journal of Youth Adolescence, 36, 583-592.

Bancroft, J. (2006). Normal sexual development. In H.E. Barbaree \& W.L. Marshall (Eds.), The Juvenile Sex Offender (pp. 19-57). New York: The Guilford Press. Barbaree, H.E. \& Langton, C.M. (2006). The effects of child sexual abuse and family environment. In H. E. Barbaree \& W. L. Marshall (Eds.), The Juvenile Sex 
Offender (Vol. 2) (pp. 58-76). New York: The Guilford Press.

Barbaree, H.E., Hudson, S.M., \& Seto, M.C. (1993). Sexual assault in society: The role of the juvenile offender. In H.E. Barbaree, W.L. Marshall, \& S.M. Hudson (Eds.), The Juvenile Sex Offender (pp. 1-24). New York: The Guildford Press.

Barbaree, H.E. \& Marshall, W.L. (2006). An introduction to the juvenile sexual offender: Terms, concepts, and definitions. In H. E. M. Barbaree, W.L. Marshall (Eds.), The Juvenile Sex Offender (pp. pp.1-18). New York: The Guilford Press.

Bardis, P.D. (1959). A familism scale. Marriage and Family Living, 21(4), 340-341.

Baron, R.M., \& Kenny, D.A. (1986). The moderator-mediator variable distinction in social psychological research: Conceptual, strategic, and statistical considerations. Journal of Personality and Social Psychology, 51(6), 1173-1182.

Becker, J.V. \& Abel, G.G. (1985). Methodological and ethical issues in evaluating and treating adolescent sexual offenders. In E.M. Otey \& G.D. Ryan (Eds.), Adolescent sex offenders: Issues in research and treatment (pp. 109-129). Rockville, MD: U.S. Department of Health and Human Services.

Berry, J.W. (2001). A psychology of immigration. Journal of Social Issues, 57(3), 615631.

Berry, J.W. (2002). Conceptual approaches to acculturation. In K.M. Chun, P. Balls Organista, \& G. Marín (Eds.), Acculturation: Advances in theory, measurement, and applied research (pp. 17-37). Washington, D.C.: American Psychological Association.

Birman, D. (1998). Biculturalism and perceived competence of Latino immigrant adolescents. American Journal of Community Psychology, 26(3), 335-354. 
Bischof, G.P., Stith, S.M., Wilson, S.M. (1992). A comparison of the family systems of adolescent sexual offenders and nonsexual offending delinquents. Family Relations, 41(318-323).

Bischof, G.P. \& Stith, S.M. (1995). Family environments of adolescent sex offenders and other juvenile delinquents. Adolescence, 30(117), 157-171.

Brook, J. S., Whiteman, M., Balka, E.B., \& Cohen, P. (1997). Drug use and delinquency: Shared and unshared risk factors in African American and Puerto Rican adolescents. The Journal of Genetic Psychology, 158(1), 25-39.

Bukowski, W.M., Sippola, L., \& Brender, W. (1993). Where does sexuality come from?: Normative sexuality from a development perspective. In H.E. Barbaree, W.L. Marshall, \& S.M. Hudson (Eds.), The Juvenile Sex Offender (pp. 84-103). New York: The Guilford Press.

Bureau of Justice Statistics. Criminal Offender Statistics. U.S. Department of Justice. Retrieved 17 February, 2009. From http://www.ojp.usdoj.gov/bjs/crimoff.htm. Buriel, R., Calzada, S., Vasquez, R. (1982). The relationship of traditional Mexican American culture to adjustment and deliquency among three generations of Mexican American male adolescents. Hispanic Journal of Behavioral Sciences, $4(1), 41-55$.

Cachelin, F.M., Schug, R.A., Juarez, L.C., \& Monreal, T.K. (2005). Sexual abuse and eating disorders in a community sample of Mexican American women. Hispanic Journal of Behavioral Sciences, 27(4), 533-546.

Coatsworth, J.D., Maldonado-Molina, M., Pantin, H., \& Szapocznik, J. (2005). A person-centered and ecological investigation of acculturation strategies in 
Hispanic immigrant youth. Journal of Community Psychology, 33(2), 157-174.

Coohey, C. (2001). The relationship between familism and child maltreatment in Latino and Anglo families. Child Maltreatment, 6, 130-142.

Cuéllar, I., Nyberg, B., Maldonado, E.R., \& Roberts, R.E. (1997). Ethnic identity and acculturation in a young adult Mexican-origin population. Journal of Community Psychology, 25(6), 535-549.

Dahl, R.E. (2004). Adolescent brain development: A period of vulnerabilities and opportunities. Annals of New York Academy of Science, 1021, 1-22.

Davis, G.E. \& Leitenberg, H. (1987). Adolescent sex offenders. Psychological Bulletin, 101(3), 417-427.

Elliott, D. S., Huizinga, D., \& Morse, B.J. (1985). The dynamics of deviant behavior: A national survey progress report. National Institute of Mental Health, Department of Health and Human Services.

Finch, B.K. \& Vega, W.A. (2003). Acculturation stress, social support, and self-rated health among Latinos in California. Journal of Immigrant Health, 5(3), 109-117.

Finkelhor, D. (1994). Current information on the scope and nature of child sexual abuse. The Future of Children, 4(2), 31-53.

Finkelhor, D. \& Browne, A. (1985). The traumatic impact of child sexual abuse: A conceptualization. American Journal of Orthopsychiatry, 55(4), 530-543.

Fontes, L.A., Cruz, M., \& Tabachnick, J. (2001). View of child sexual abuse in two cultural communities: An exploratory study among African Americans and Latinos. Child Maltreatment, 6(2), 103-117.

Fontes, L.A. (2007). Sin vergüenza: Addressing shame with Latino victims of child 
sexual abuse and their families. Journal of Child Sexual Abuse, 16(1), 61-83.

Fridrich, A.H. \& Flannery, D.J. (1995). The effects of ethnicity and acculturation on early adolescent delinquency. Journal of Child and Family Studies, 4(1), 69-87.

Friedrich, W.N. \& Luecke, W.L. (1988). Young school-age sexually aggressive children. Professional Psychology: Research and Practice, 19(2), 155-164.

Gaines, S.O., Marelich, W.D., Bledsoe, K.L., Steers, W.N., Henderson, M.C., Granrose, C.S., Barájas, L., Hicks, D., Lyde, M., Takahashi, Y., Yum, N., Ríos, D.I., García, B.F., Farris, K.R., \& Page, M.S. (1997). Links between race/ethnicity and cultural values as mediated by racial/ethnic identity and moderated by gender. Journal of Personality and Social Psychology, 72(6), 1460-1476.

Gamst, G., Dana, R.H., Der-Karabetian, A., Aragón, M., Arrellano, L.M., \& Kramer, T. (2002). Effects of Latino acculturation and ethnic identity on mental health outcomes. Hispanic Journal of Behavioral Sciences, 24(4), 479-504.

Gil, A.G., Vega, W.A., \& Biafora, F. (1998). Temporal influences of family structure and family risk factors on druguse initiation in a multiethnic sample of adolescent boys. Journal of Youth and Adolescence, 27(3), 373-393.

Gil, A.G., Wagner, E.F., \& Vega, W.A. (2000). Acculturation, familism, and alcohol use among Latino adolescent males: Longitudinal relations. Journal of Community Psychology, 28(4), 443-458.

Gil, A.G. \& Vega, W.A. (1996). Two different worlds: Acculturation stress and adaptation among Cuban and Nicaraguan families. Journal of Social and Personal Relationships, 13(3), 435-456.

Gorman-Smith, D., Tolan, P.H., Zelli, A., \& Huesmann, L.R. (1996). The relation of 
family functioning to violence among inner-city minority youths. Journal of Family Psychology, 10(2), 115-129.

Gray, A., Bucsoni, A., Houchens, P., \& Pithers, W.D. (1997). Children with sexual behavior problems and their caregivers: Demographics, functioning, and clinical patterns. Sexual Abuse: A Journal of Research and Treatment, 9(4), 267-290.

Gray, A., Pithers, W.D., Busconi, A., \& Houchens, P. (1999). Development and etiological characteristics of children with sexual behavior problems: Treatment implications. Child Abuse \& Neglect, 23(6), 601-621.

Groth, A. N. \& Loredo, C.M. (1981). Juvenile sexual offenders: Guidelines for assessment. International Journal of Offender Therapy and Comparative Criminology, 25, 31-39.

Heller, P.L. (1970). Familism scale: A measure of family solidarity. Journal of Marriage and the Family, 32(1), 73-80.

Heller, P.L. (1976). Familism scale: Revalidation and revision. Journal of Marriage and the Family, 38(3), 423-429.

Hinson, J.V., Koverola, C., \& Morahan, M. (2002). An empirical investigation of the psychological sequelae of childhood sexual abuse in an adult Latina population. Violence Against Women, 8(7), 816-844.

Hovey, J.D. \& King, C.A. (1996). Acculturative stress, depression, and suicidal ideation among immigrant and second-generation Latino adolescents. Journal of American Academy of Child and Adolescent Psychiatry, 35(9), 1183-1192.

Hu, L \& Bentler, P.M. (1999). Cutoff criteria for fit indexes in covariance structure analysis: Conventional criteria versus new alternatives. Structural Equation 
Modeling, 6(1), 1-55.

Hunter, J.A. \& Becker, J.V. (1994). The role of deviant sexual arousal in juvenile sexual offending: Etiology, evaluation, and treatment. Criminal Justice and Behavior, 21(1), 132-149.

Jacobs, W.L., Kennedy, W.A., \& Meyer, J.B. (1997). Juvenile delinquents: A betweengroup comparison study of sexual and nonsexual offenders. Sexual Abuse: A Journal of Research and Treatment, 9(3), 201-217.

Jaffe, P. G., Suderman, M., \& Reitzel, D. (1992). Working with children and adolescents to end the cycle of violence: A social learning approach to intervention and prevention programs. In R.D. Peters, R.J. McMahon, \& V.L. Quinsey (Eds.), Aggression and Violence throughout the Lifespan (pp. 83-99). London: Sage.

John, O.P. \& Benet-Martínez, V. (2000). Measurement: Reliability, construct validity, and scale construction. In H.T. Reis \& C.M. Judd, Handbook of Research: Methods in Social and Personality Psychology (pp. 412-450). New York: Cambridge University Press.

Johnson, T.C. (1988). Child perpetrators - children who molest other children: Preliminary findings. Child Abuse \& Neglect, 12, 219-229.

Johnson, T.C. (1989). Female child perpetrators: Children who molest other children. Child Abuse \& Neglect, 13, 571-585.

Jones, L. \& Finkelhor, D. (2001). The decline in child sexual abuse cases. In J.J. Wilson (Ed.) Office of Juvenile Justice and Delinquency Prevention, U.S. Department of Justice. Retrieved 13 May, 2009. From http://www.ncjrs.gov/html/ojjdp/jjbul2001_1_1/contents.html. 
Kaufman, K.L. (1994). Modus operandi questionnaire: Revised version. Columbus, OH: Author (Ohio State Children's Hospital).

Kaufman, K.L. (2001). Demographics Questionnaire. Portland, OR: Author (Portland State University).

Kaufman, K.L. (2001). Supervision Questionnaire. Portland, OR: Author (Portland State University).

Kaufman, K.L., Hilliker, D.R., Lathrop, P., \& Daleiden, E.L. (1993). Assessing child sexual offenders' modus operandi: Accuracy in self-reported use of threats and coercion. Sexual Abuse: A Journal of Research and Treatment, 6(3), 213-229.

Kaufman, K.L., Hilliker, D.R., Lathrop, P., Daleiden, E.L., \& Rudy, L. (1996). Sexual offenders' modus operandi: A comparison of structured interview and questionnaire approaches. Journal of Interpersonal Violence, 11(19), 19-34.

Keith, T.Z., Fine, J.G., Taub, G.E., Reynolds, M.R., \& Kranzler, J.H. (2006). Higher order, multisample, confirmatory factor analysis of the Wechsler Intelligence Scale for Children - Fourth Edition: What does it measure? School Psychology Review, 35(1), 108-127.

Kendall-Tackett, K.A., Williams, L.M., \& Finkelhor, D. (1993). Impact of sexual abuse on children: A review and synthesis of recent empirical studies. Psychological Bulletin, 113(1), 164-180.

Kenny, M.C. \& McEachern, A.G. (2007). Family environment in Hispanic college females with a history of childhood sexual abuse. Journal of Child Sexual Abuse, 16(3), 19-40.

Knight, R. A. \& Prentky, R.A. (1993). Exploring characteristics for classifying juvenile 
sex offenders. In H.E. Barbaree, W.L. Marshall, \& S.M. Hudson (Eds.), The Juvenile Sex Offender (pp. 45-83). New York: The Guilford Press.

Knox, L.A. (2009). Juvenile Sex Offenders: A Consideration of Attachment Deficits in the Etiology of Offending. Unpublished master's thesis, Portland State University, Portland, Oregon.

Landau, J., Cole, R.E., Tuttle, J., Clements, C.D., \& Stanton, M.D. (2000). Family connectedness and women's sexual risk behaviors: Implications for the prevention/intervention of STD/HIV infection. Family Process, 39(4), 461-475.

Langton, C.M. \& Barbaree, H.E. (2006). Conceptual issues in treatment evaluation research with juvenile sexual offenders. In H.E. Barbaree \& W.L. Marshall (Eds.), The Juvenile Sex Offender: Second Edition (pp. 248-274). New York: The Guilford Press.

Lara, M., Gamboa, C., Kahramanian, M.I., Morales, L.S., \& Hayes Bautista, D.E. (2005). Acculturation and Latino health in the United States: A review of the literature and its sociopolitical context. Annual Review of Public Health, 26, 367-397.

Lay, C., Fairlie, P., Jackson, S., Ricci, T., Eisenberg, J., Sato, T., Teeaar, A., \& Melamud, A. (1998). Domain-specific allocentrism-idiocentrism: A measure of family connectedness. Journal of Cross-Cultural Psychology, 29(3), 434-460.

Marín, G. \& Gamba, R.J. (2002). Acculturation and changes in cultural values. In K.M. Chun, P. Balls Organista, \& G. Marín (Eds.), Acculturation: Advances in theory, measurement, and applied research (pp. 83-93). Washington, D.C.: American Psychological Association.

Marín, G., Sabogal, F., VanOss Marín, B., Otero-Sabogal, R., \& Perez-Stable, E.J., 
(1987). Development of a short acculturation scale for Hispanics. Hispanic Journal of Behavioral Sciences, 9(2), 183-205.

Markham, C.M., Tortolero, S., Escobar-Chaves, L., Parcel, G.S., Harrist, R., \& Addy, R.C. (2003). Family connectedness and sexual risk-taking among urban youth attending alternative schools. Perspectives on Sexual and Reproductive Health, 35(4), 174-179.

Marshall, W.L. (1993). The role of attachments, intimacy, and loneliness in the etiology and maintenance of sexual offending. Sexual and Marital Therapy, 8(2), 109121.

Marshall, W. L. Marshall, L.E. (2000). The origins of sexual offending. Trauma, Violence, \& Abuse, 1(3), 250-263.

Marshall, W. L. Mazucco, A. (1995). Self-esteem and parental attachments in child molesters. Sexual Abuse: A Journal of Research and Treatment, 7(4), 279-285.

McDonald, R.P. (1999). Test theory: A unified treatment. Mahwah, NJ: Lawrence Erlbaum Associates, Inc.

Meyerson, L.A., Long. P.J., Miranda, R., \& Marx, B.P. (2002). The influence of childhood sexual abuse, physical abuse, family environment, and gender on the psychological adjustment of adolescents. Child Abuse \& Neglect, 26, 387-405.

Miller, K.S., Forehand, R., \& Kotchick, B.A. (1999). Adolescent sexual behavior in two ethnic minority samples: The role of family variables. Journal of Marriage and Family, 61(1), 85-98.

Milloy, C.D. (1994). A comparative study of juvenile sex offenders and non-sex offenders. Washington State Institute for Public Policy. 
Mio, J.S., Barker, L.A., \& Tumambing, J. (2009). Multicultural Psychology: Understanding our diverse communities ( $2^{\text {nd }}$ edition), New York: McGraw Hill.

Miranda, A.O., Estrada, D., \& Firpo-Jimenez, M. (2000). Family cohesion, adaptability, and environment among Latino families in dissimilar stages of acculturation. The Family Journal, 8(4), 341-350.

Murphy, G. (2002). Beyond surviving: Toward a movement to prevent child sexual abuse. M.S. Foundation for Women. Retrieved 20 May, 2009. From http://www.ms.foundation.org/user-assets/PDF/Program/safety.pdf.

Norris, A.E., Ford, K., \& Bova, C.A. (1996). Psychometrics of a brief acculturation scale for Hispanics in a probability sample of urban Hispanic adolescents and young adults. Hispanic Journal of Behavioral Sciences, 18(1), 29-38.

Paolucci, E.O., Genuis, M.L., \& Violato, C. (2001). A meta-analysis of the published research on the effects of child sexual abuse. The Journal of Psychology, 135(1), 17-36.

Patterson, L.B., Valenzuela, S.J., Hayes, L.A., Yagoda, J., \& Kaufman, K.L. (2009). I'm watching you: Parental supervision \& ethnicity in juvenile sex offending. Poster presented at the Association for the Treatment of Sexual Abusers (ATSA). Dallas, TX: 30 September, 2009.

Phinney, J.S. (1992). The Multigroup Ethnic Identity Measure: A new scale for use with diverse groups. Journal of Adolescent Research, 7(2), 156-176.

Pithers, W. D., Gray, A., Busconi, A., \& Houchens, P. (1998). Caregivers of children with sexual behavior problems: Psychological and familial functioning. Child Abuse \& Neglect, 22(2), 129-141. 
Pithers, W. D. \& Gray, A. (1998). The other half of the story: Children with sexual behavior problems. Psychology, Public Policy, and Law, 4(1/2), 200-217.

Portes, A. \& Rumbaut, R.G. (2001). Not everyone is chosen: Segmented assimilation and its determinants. In A. Portes \& R.G. Rumbaut, Legacies: The story of the immigrant second generation (pp. 44-69). Los Angeles: University of California Press.

Portes, A. \& Zhou, M. (1993). The new second generation: Segmented assimilation and its variants. Annals of the American Academy of Political and Social Science, 530, 74-96.

Rapkin, B.D. \& Luke, D.A. (1993). Cluster analysis in community research: Epistemology and practice. American Journal of Community Psychology, 21(1), 247-277.

Rappaport, J. (1977). What is Community Psychology and where does it come from? Community Psychology: Values, research, and action (pp. 1-53). New York: Holt, Rheinhart, \& Winston.

Rappaport, J. (2005). Community Psychology is (thank God) more than science. American Journal of Community Psychology, 35(3/4), 231-238.

Rodriguez, N., Mira, C.B., Paez, N.D., \& Myers, H.F. (2007). Exploring the complexities of familism and acculturation: Central constructs for people of Mexican origin. American Journal of Community Psychology, 39, 61-77.

Rodriguez, O. \& Weisburd, D. (1991). The integrated social control model and ethnicity: The case of Puerto Rican American delinquency. Criminal Justice and Behavior, $18,464-479$. 
Romero, A.J., Robinson, T.N., Haydel, K.F., Mendoza, F., \& Killen, J.D. (2004). Associations among familism, language usage, and education in MexicanAmerican mothers and their children. Developmental and Behavioral Pediatrics, 25(1), 34-40.

Rumbaut, R.G. (1994). The crucible within: Ethnic identity, self-esteem, and segmented assimilation among children of immigrants. International Migration Review, 28(4), 748-794.

Ryan, G., Lane, S., Davis, J., Isaac, C. (1987). Juvenile sex offenders: Development and correction. Child Abuse \& Neglect, 11, 385-395.

Sabogal, F., Marín, G., Otero-Sabogal, R., Marín, B.V., \& Perez-Stable, E.J. (1987). Hispanic familism and acculturation: What changes and what doesn't? Hispanic Journal of Behavioral Sciences, 9(4), 397-412.

Samaniego, R.Y. \& Gonzales, N.A. (1999). Multiple mediators of the effects of acculturation status on delinquency for Mexican American adolescents. American Journal of Community Psychology, 27(2), 189-210.

Sanders-Phillips, K., Moisan, P.A. Wadlington, S., Morgan, S., \& English, K. (1995). Ethnic differences in psychological functioning among Black and Latino sexually abused girls. Child Abuse \& Neglect, 19(6), 691-706.

Sarkisian, N., Gerena, M., \& Gerstel, N. (2006). Extended family ties among Mexicans, Puerto Ricans, and Whites: Superintegration or disintegration? Family Relations, $55,331-344$.

Sarkisian, N., Gerena, M., \& Gerstel, N. (2007). Extended family integration among Euro and Mexican Americans: Ethnicity, gender, and class. Journal of Marriage 
and Family, 69, 40-54.

Schecter, D.S., Brunelli, S.A., Cunningham, N., Brown, J., \& Baca, P. (2002). Motherdaughter relationships and child sexual abuse: A pilot study of 35 dyads. Bulletin of the Menninger Clinic, 66(1), 39-60.

Schwartz, S.J., Pantin, H., Prado, G., Sullivan, S., \& Szapocznik, J. (2005). Family functioning, identity, and problem behavior in Hispanic immigrant early adolescnts. Journal of Early Adolescence, 25(4), 392-420.

Sefarbi, R. (1990). Admitters and deniers among adolescent sex offenders and their families: A preliminary study. American Journal of Orthopsychiatry, 60(3), 460465.

Smallbone, S.W. (2006). Social and psychological factors in the development of delinquency and sexual deviance. In H.E. Barbaree \& W.L. Marshall (Eds.), The Juvenile Sex Offender (pp. 105-127). New York: The Guilford Press.

Starzyk, K.B. \& Marshall, W.L. (2003). Childhood family and personological risk factors for sexual offending. Aggression and Violent Behavior, 8, 93-105.

Steidel, A.G.L. \& Contreras, J.M. (2003). A new familism scale for use with Latino populations. Hispanic Journal of Behavioral Sciences, 25(3), 312-330.

Sullivan, S., Schwartz, S.J., Prado, G., Huang, S., Pantin, H., Szapocznik, J. (2007). A bidimensional model of acculturation for examining differences in family functioning and behavior problems in Hispanic immigrant adolescents. Journal of Early Adolescence, 27(4), 405-430.

Taylor, D.M. \& Lambert, W.E. (1996). The meaning of multiculturalism in a culturally diverse urban American area. The Journal of Social Psychology, 136(6), 727- 
740.

Trickett, E.J. (1996). A future for Community Psychology: The contexts of diversity and the diversity of contexts. American Journal of Community Psychology, 24(2), 209-233.

Ullman, S. \& Filipas, H.H. (2005). Ethnicity and child sexual abuse experiences of female college students. Journal of Child Sexual Abuse, 14(3), 67-89.

U.S. Census Bureau (2009). Population Estimates. Retrieved 5 January, 2010. From http://www.census.gov.

U.S. Department of Health \& Human Services (2006). Child Maltreatment 2006.

Retrieved 25 March, 2009. From

http://www.acf.hhs.gov/programs/cb/stats_research/index.htm\#can.

Valenzuela, A. \& Dornbusch, S.M. (1994). Familism and social capital in the academic achievement of Mexican origin and Anglo Adolescents. Social Science Quarterly, 75(1), 18-36.

Vega, W.A., Gil, A.G., Warheit, G.J., Zimmerman, R.S.. \& Apospori, E. (1993). Acculturation and delinquent behavior among Cuban American adolescents: Toward and empirical model. American Journal of Community Psychology, 21, 113-125.

Veneziano, C. \& Veneziano, L. (2002). Adolescent sex offenders: A review of the literature. Trauma, Violence, \& Abuse, 3(4), 247-260.

Villareal, R., Blozis, S.A., \& Widaman, K.F. (2005). Factorial invariance of a panHispanic familism scale. Hispanic Journal of Behavioral Sciences, 27(4), 409425. 
Wall, J.A., Power, T.G., \& Arbona, C. (1993). Susceptibility to antisocial peer pressure and its relation to acculturation in Mexican-American adolescents. Journal of Adolescent Research, 8(4), 403-418.

Watts, R.J. (1992). Elements of a psychology of human diversity. Journal of Community Psychology, 20, 116-131.

Wegener, D.T. \& Fabrigar, L.E. (2000). Analysis and design for nonexperimental data: Addressing causal and noncausal hypotheses. In H.T. Reis \& C.M. Judd, Handbook of Research: Methods in Social and Personality Psychology (pp. 412450). New York: Cambridge University Press.

Zambrana, R.E., Silva-Palacios, V., Powell, D. (1992). Parenting concerns, family support systems, and life problems in Mexican-origin women: A comparison by nativity. Journal of Community Psychology, 20, 276-288. 
Appendix A.

Time Periods

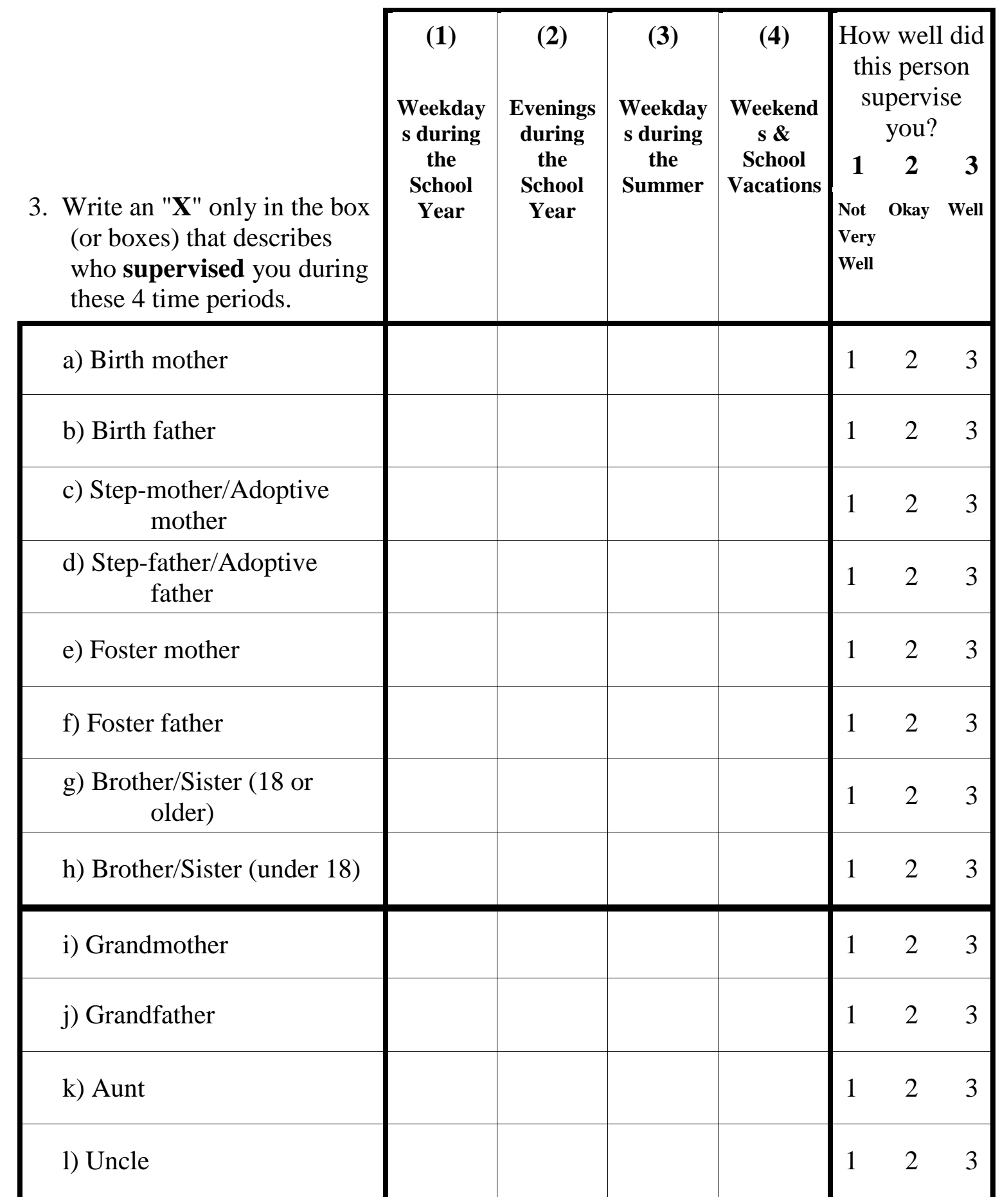




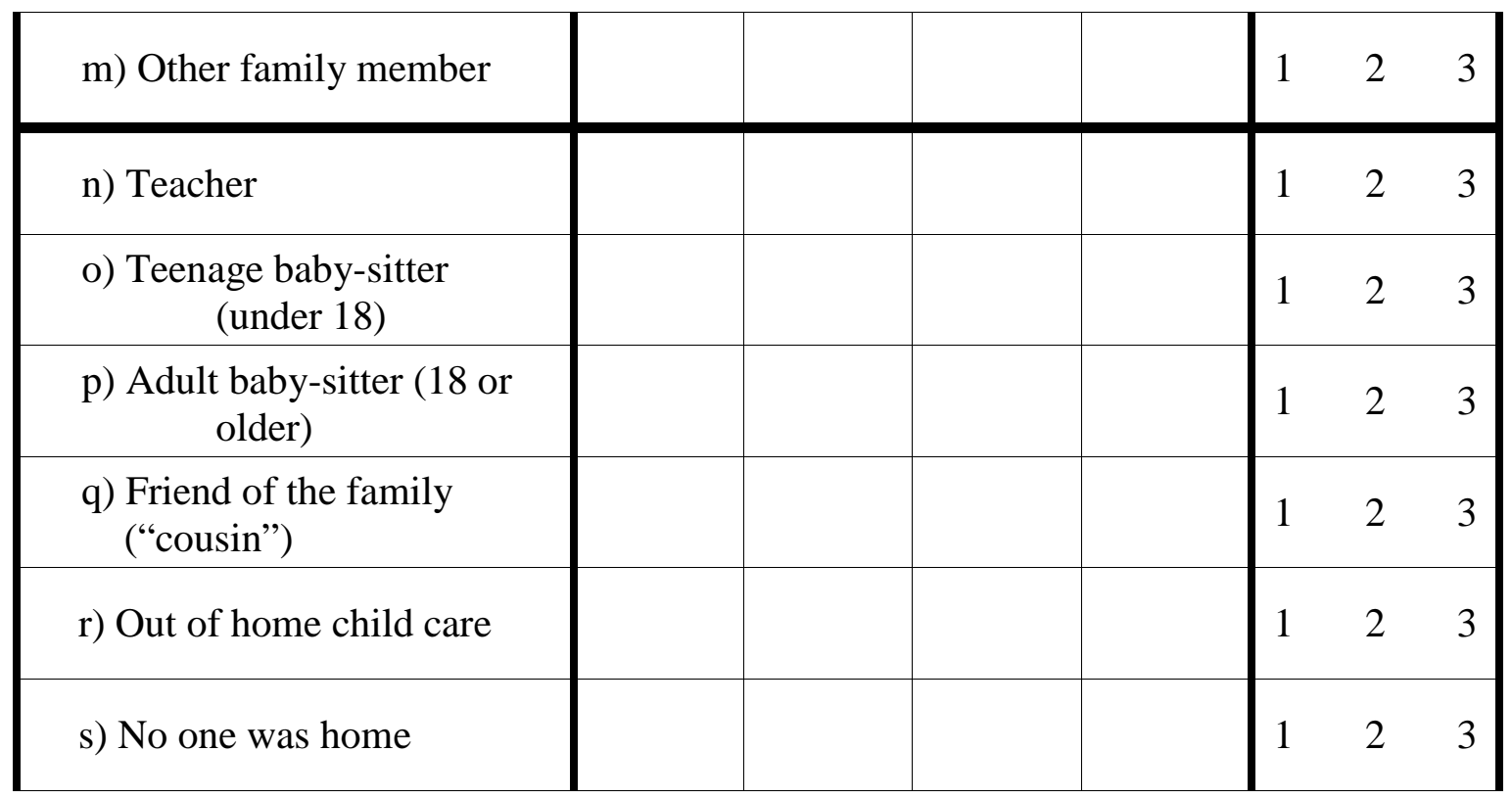


Appendix B.

$\begin{array}{ccccc}0 & 1 & 2 & 3 & 4 \\ \text { Never } & \text { Almost Never } & \text { Sometimes } & \text { Almost Always } & \text { Always }\end{array}$

30. How often did you do these activities with your supervisor?

\begin{tabular}{|l|llcc|}
\hline a) My supervisor and I did activities together (like played games). & 0 & 1 & 3 \\
\hline $\begin{array}{l}\text { b) My supervisor went to my activities (like watched me play } \\
\text { sports). }\end{array}$ & 0 & 1 & 2 & 3 \\
\hline c) My supervisor taught me things (like how to cook). & 0 & 1 & 2 & 3 \\
\hline d) My supervisor helped me with my homework. & 0 & 1 & 2 & 3 \\
\hline e) We ate our meals together. & 0 & 1 & 2 & 3 \\
\hline f) We went to the park together. & 0 & 1 & 2 & 3 \\
\hline g) We went to church together. & 0 & 1 & 2 & 3 \\
\hline
\end{tabular}


Appendix C.

0

Never

32. How often did you talk with your supervisor about:

Almost Never

Sometimes

Almost Always

\begin{tabular}{|llllll}
\hline 0 & 1 & 2 & 3 & 4
\end{tabular}

Who usually started

the conversation?

(Circle one.)

ME MY

SUPERVISOR

a) your school work?

0

0

2

$3-4$

b) your behavior at school?

c) other things at school?

Like what?

d) your behavior at home?

e) your friends?

f) dating relationships?

h) family issues?

Like what?

\begin{tabular}{l}
\hline i) chores? \\
\hline $\begin{array}{l}\text { j) something good that } \\
\text { happened? }\end{array}$ \\
\hline k) something bad that happened?
\end{tabular}

1) your life?

m) your supervisor's life?

$\begin{array}{llll}0 & 1 & 2 & 3\end{array}$

ME MY SUPERVISOR

ME MY SUPERVISOR

ME MY SUPERVISOR

ME MY SUPERVISOR

ME MY SUPERVISOR

ME MY SUPERVISOR

ME MY SUPERVISOR

ME MY SUPERVISOR

ME MY SUPERVISOR

ME MY SUPERVISOR

ME MY SUPERVISOR

ME MY SUPERVISOR 


\begin{tabular}{|l|llllll|l|}
\hline n) drugs or alcohol? & 0 & 1 & 2 & 3 & 4 & $\begin{array}{c}\text { ME MY } \\
\text { SUPERVISOR }\end{array}$ \\
\hline
\end{tabular}


Appendix D.

$\begin{array}{ccccc}0 & 1 & 2 & 3 & 4 \\ \text { Never } & \text { Almost Never } & \text { Sometimes } & \text { Almost Always } & \text { Always }\end{array}$

31. How often were the following statements true about the relationship you had with your supervisor?

\begin{tabular}{|l|lllll|}
\hline a) My supervisor trusted me. & 0 & 1 & 2 & 3 & 4 \\
\hline b) My supervisor accepted me for who I was. & 0 & 1 & 2 & 3 & 4 \\
\hline c) My supervisor expected me to do the "right thing." & 0 & 1 & 2 & 3 & 4 \\
\hline d) My supervisor understood where I was coming from. & 0 & 1 & 2 & 3 & 4 \\
\hline e) My supervisor asked for my opinion about things. & 0 & 1 & 2 & 3 & 4 \\
\hline f) I talked to my supervisor about personal things. & 0 & 1 & 2 & 3 & 4 \\
\hline
\end{tabular}


Appendix E.

\section{4 Strongly disagree Somewhat disagree \\ Somewhat agree Strongly agree}

\begin{tabular}{|c|c|c|c|c|}
\hline $\begin{array}{l}\text { B-7 I have spent time trying to find out more about my own ethnic group, } \\
\text { such as its history, traditions, and customs. }\end{array}$ & 4 & 3 & 2 & 1 \\
\hline $\begin{array}{l}\text { B-8 I am active in organizations or social groups that include mostly } \\
\text { members of my own ethnic group. }\end{array}$ & 4 & 3 & 2 & 1 \\
\hline $\begin{array}{l}\text { B-9 I have a clear sense of my ethnic background and what it means for } \\
\text { me. }\end{array}$ & 4 & 3 & 2 & 1 \\
\hline $\begin{array}{l}\text { B-10 I like meeting and getting to know people from ethnic groups other } \\
\text { than my own. }\end{array}$ & 4 & 3 & 2 & 1 \\
\hline $\begin{array}{l}\text { B-11 I think a lot about how my life will be affected by my ethnic } \\
\text { background. }\end{array}$ & 4 & 3 & 2 & 1 \\
\hline B-12 I am happy that I am a member of the group I belong to. & 4 & 3 & 2 & 1 \\
\hline $\begin{array}{l}\text { B-13 I sometimes feel it would be better if different ethnic groups didn't } \\
\text { try to mix together. }\end{array}$ & 4 & 3 & 2 & 1 \\
\hline B-14 I am not very clear about the role of my ethnicity in my life. & 4 & 3 & 2 & 1 \\
\hline $\begin{array}{l}\text { B-15 I often spend time with people from ethnic groups other than my } \\
\text { own. }\end{array}$ & 4 & 3 & 2 & 1 \\
\hline $\begin{array}{l}\text { B-16 I have not spent much time trying to learn more about the culture and } \\
\text { history of my own ethnic group. }\end{array}$ & 4 & 3 & 2 & 1 \\
\hline B-17 I have a strong sense of belonging to my own ethnic group. & 4 & 3 & 2 & 1 \\
\hline $\begin{array}{l}\text { B-18 I understand what my ethnic background means to me, in terms of } \\
\text { how to relate to my own group and other groups. }\end{array}$ & 4 & 3 & 2 & 1 \\
\hline $\begin{array}{l}\text { B-19 In order to learn more about my ethnic background, I have often } \\
\text { talked to other people about my ethnic group. }\end{array}$ & 4 & 3 & 2 & 1 \\
\hline B-20 I have a lot of pride in my ethnic group and its accomplishments. & 4 & 3 & 2 & 1 \\
\hline B-21 I don't try to become friends with people from other ethnic groups. & 4 & 3 & 2 & 1 \\
\hline $\begin{array}{l}\text { B-22 I participate in cultural practices of my own group, such as special } \\
\text { food, music, or other customs. }\end{array}$ & 4 & 3 & 2 & 1 \\
\hline B-23 I am involved in activities with people from other ethnic groups. & 4 & 3 & 2 & 1 \\
\hline B-24 I feel a strong attachme & 4 & 3 & 2 & 1 \\
\hline
\end{tabular}




\begin{tabular}{|l|llrr|}
\hline B-25 I enjoy being around people from ethnic groups other than my own. & 4 & 3 & 2 & 1 \\
\hline B-26 I feel good about my cultural or ethnic background. & 4 & 3 & 2 & 1 \\
\hline
\end{tabular}


Appendix F.

0

Never
1

Almost Never
2

Sometimes
3

Almost
4

Always

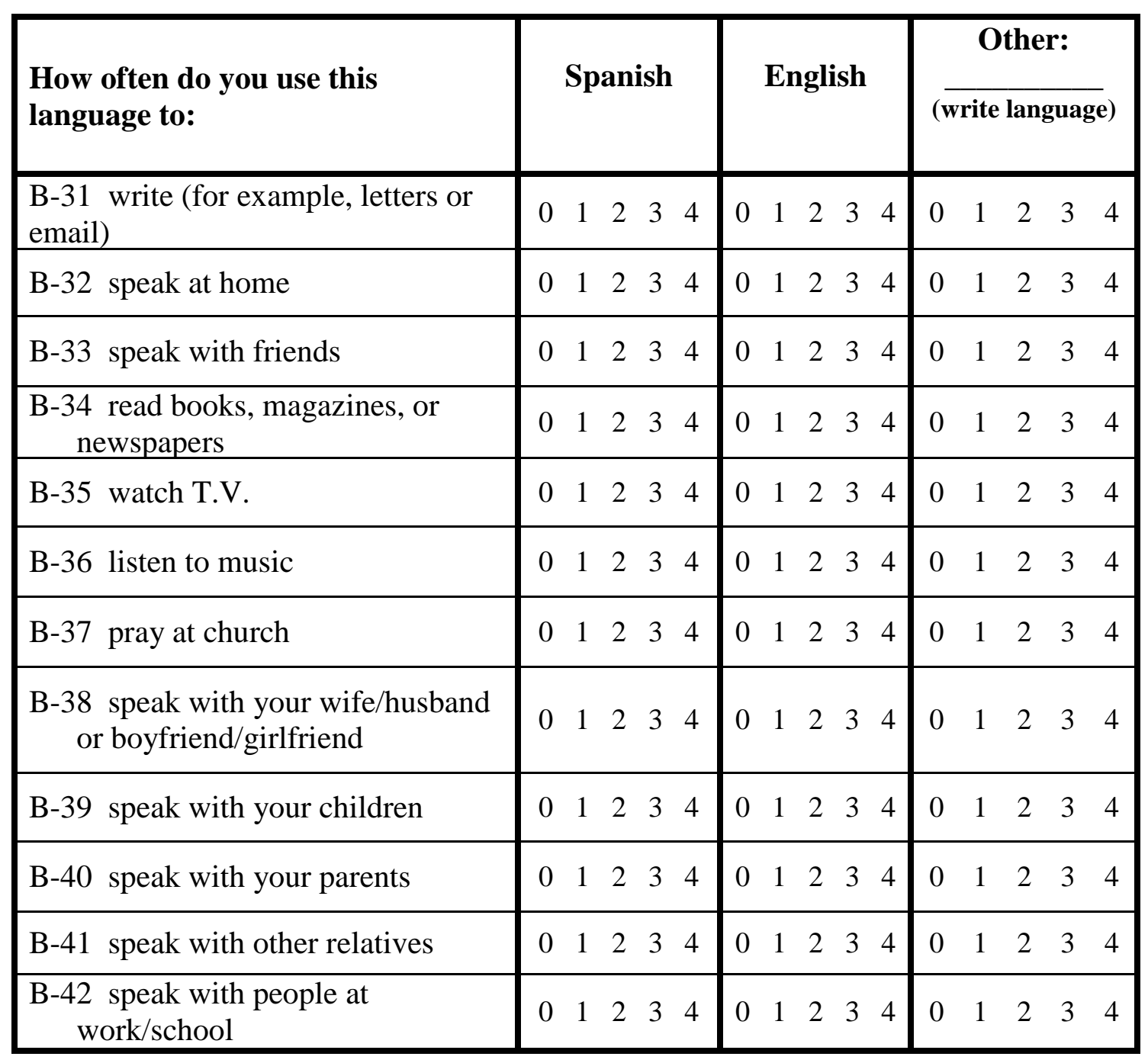

